\title{
Tomography of the positive-pitch fast-ion velocity distribution in DIII-D plasmas with Alfvén eigenmodes and neoclassical tearing modes
}

Madsen, B.; Salewski, M.; Heidbrink, W.W.; Stagner, L.; Podestà, M.; Lin, D.; Garcia, A.V.; Hansen, Per Christian; Huang, J.

Published in:

Nuclear Fusion

Link to article, DOI:

10.1088/1741-4326/ab82b5

Publication date:

2020

Document Version

Early version, also known as pre-print

Link back to DTU Orbit

Citation (APA):

Madsen, B., Salewski, M., Heidbrink, W. W., Stagner, L., Podestà, M., Lin, D., Garcia, A. V., Hansen, P. C., \& Huang, J. (2020). Tomography of the positive-pitch fast-ion velocity distribution in DIII-D plasmas with Alfvén eigenmodes and neoclassical tearing modes. Nuclear Fusion, 60(6), [066024 ]. https://doi.org/10.1088/1741$4326 / a b 82 b 5$

\section{General rights}

Copyright and moral rights for the publications made accessible in the public portal are retained by the authors and/or other copyright owners and it is a condition of accessing publications that users recognise and abide by the legal requirements associated with these rights.

- Users may download and print one copy of any publication from the public portal for the purpose of private study or research.

- You may not further distribute the material or use it for any profit-making activity or commercial gain

- You may freely distribute the URL identifying the publication in the public portal 


\title{
Tomography of the positive-pitch fast-ion velocity distribution in DIII-D plasmas with Alfvén eigenmodes and neoclassical tearing modes
}

\author{
B. Madsen ${ }^{1}$, M. Salewski ${ }^{1}$, W. W. Heidbrink ${ }^{2}$, L. Stagner ${ }^{2}$, \\ M. Podestà ${ }^{3}$, D. Lin ${ }^{2}$, A. V. Garcia ${ }^{2}$, P. C. Hansen ${ }^{4}$, \\ J. Huang ${ }^{5}$ and the DIII-D team \\ 1 Department of Physics, Technical University of Denmark, Kgs. Lyngby, \\ Denmark \\ 2 Department of Physics and Astronomy, University of California Irvine, Irvine, \\ CA, USA \\ 3 Princeton Plasma Physics Laboratory, Princeton, NJ, USA \\ 4 Department of Applied Mathematics and Computer Science, Technical \\ University of Denmark, Kgs. Lyngby, Denmark \\ 5 Institute of Plasma Physics, Chinese Academy of Sciences, P.O. 1126, 230031 \\ Hefei, Anhui, China \\ E-mail: birma@fysik.dtu.dk \\ February 2020
}

\begin{abstract}
.
Understanding the effect of Alfvén eigenmodes (AEs) and neoclassical tearing modes (NTMs) on fast ions is highly important for fusion reactors due to potentially strong resonant interactions between the fast ions and the modes. Here, we use the four-view fast-ion D-alpha (FIDA) diagnostic installed in the DIII-D tokamak to reconstruct the fast-ion velocity distribution at two radial positions during two sequential discharges with strong and weak mode activity, respectively. The velocity-space coverage of the diagnostics, however, only allows reliable reconstructions of fast ions with positive pitches. Therefore, we suggest new tomographic inversion methods relying on prior information outside the well-diagnosed region. We find that within the population of fast ions with positive pitches, ions at all energies are transported away from the measurement volumes. Comparisons between the reconstructions and kick model simulations, where the mode activity is considered, reveals that low-frequency modes such as the NTMs and low-frequency AEs contribute significantly to the positivepitch fast-ion transport in the central measurement volume, whereas TAEs and EAEs become important farther out and are responsible for decreased fast-ion confinement.
\end{abstract}

Keywords: velocity-space tomography, fast-ion D-alpha spectroscopy, fast ions, Alfvén eigenmodes, neoclassical tearing modes, kick model, DIII-D tokamak 


\section{Introduction}

In magnetic confinement fusion reactors, fast ions born in fusion reactions or generated by auxiliary heating can excite unstable Alfvén eigenmodes (AEs) that in turn might cause redistribution or losses of the fast ions $1-6$. Since fast-ion losses cause a reduction of the plasma heating efficiency and can potentially damage the plasma-facing components of the reactor 7], information about the fast-ion transport during degraded fast-ion confinement is of utmost importance.

By combining measurements from multiple fastion diagnostics, the fast-ion velocity distribution can be reconstructed by tomographic inversion. Fast-ion velocity-space tomography 8 11 has proven a useful method to study the redistribution of fast ions due to sawtooth crashes that were found to affect different regions in velocity space differently 12 17].

Previous studies on AE activity in the DIII-D tokamak have indicated that above a threshold, the transport of fast ions caused by $\mathrm{AE}$ activity is also strongly selective in velocity space 18 20]. Here, we employ fast-ion D-alpha (FIDA) measurements in the sequential discharges \#153071 and \#153072 studied in 20 21 to do the first tomographic reconstructions of the 2D fast-ion velocity distribution in the central DIII$\mathrm{D}$ plasma. In both discharges, AE activity and lowfrequency mode activity such as neoclassical tearing modes (NTM) are observed, but with vastly different amplitudes. We compare the reconstructions of the fast-ion distributions to kick model simulations 22 24] where the AE- and NTM-induced transport of the fast ions is included in the modelling of the distribution.

Each FIDA view is sensitive to a particular portion of velocity space. Reliable reconstructions of the fastion velocity distribution can be obtained in regions covered by multiple views 25. The DIII-D FIDA diagnostic only provides the necessary velocity-space coverage of more than one view in parts of velocity space. Hence, reliable reconstructions of the fast-ion distribution are not expected for all velocities. To nevertheless compute inversions in the well-diagnosed part of velocity space, we here suggest the inclusion of additional prior information in the sparsely diagnosed region and introduce new methods for reconstructing the distribution in only a restricted part of velocity space.

This paper is structured as follows. Section 2 describes the FIDA diagnostics in the DIII-D tokamak along with the experimental measurements and conditions during the considered discharges. Tomographic inversion methods for reconstructing the fast-ion distribution in all of velocity space are presented in section 3 . Section 4 introduces methods for reconstructing the velocity distribution in only a restricted part of velocity space. In section 5, these methods are tested on synthetic signals in order to evaluate their reliability when used to reconstruct a realistic, known fast-ion distribution, and employed to reconstruct the distribution from measurements during the two experimental discharges. The fast-ion velocity distribution obtained from measurements are compared to kick model distributions in section 6. Section 7 concludes the paper.

\section{Experimental setup and measurements}

The FIDA diagnostic measures the Doppler-shifted light emitted due to the $3 \rightarrow 2$ transition in excited neutrals formed from fast ions after charge exchange 26 . The deuterium ions receive electrons from deuterium neutrals injected into the plasma by neutral beam injection (NBI). Hence, the measurement volume of the FIDA diagnostics is the intersection between the FIDA line-of-sight and the neutral beam. In the DIII-D tokamak, three neutral beams are intersected by the lines-of-sight of four FIDA diagnostics at different toroidal and radial positions 20, 27, 28]. Due to the approximate toroidal symmetry of the tokamak, volumes at the same radial and vertical coordinates $(R, z)$, but different toroidal positions, are expected to have similar fastion populations. Therefore, measurements at the same $(R, z)$-coordinates from the four diagnostics can be combined in tomographic reconstructions of the fast-ion velocity distribution. This is different from previous tomographic reconstructions in velocity space at the ASDEX Upgrade $10,13,14,29$ and MAST [16 tokamaks where all lines-of-sight intersect the same neutral beam and have approximately the same measurement volume in position space. The assumption of symmetry was also used at JET [30] and will be necessary at ITER 31].

Here, we reconstruct the fast-ion distribution at two radial positions, both at $R \approx 190 \mathrm{~cm}$, i.e. $\sim$ $10 \mathrm{~cm}$ from the magnetic axis, and farther out at $R \approx 203 \mathrm{~cm}$. Both locations are observed by four FIDA views, each with spatial resolutions of $\sim 2-6 \mathrm{~cm} 20$. At $R \approx 190 \mathrm{~cm}$, the measurement volumes of the 
four views intersect almost perfectly. However, for the grouping at $R \approx 203 \mathrm{~cm}$, the centroids deviate by as much as $3.7 \mathrm{~cm}$. Due to this and greater fast-ion densities, hence better signal-to-noise ratios, closer to the magnetic axis, reconstructions of the fast-ion velocity distribution are the most reliable for $R \approx 190 \mathrm{~cm}$. This location is, therefore, employed as our reference location in this and following sections. In section 5.1 and 6, the findings at $R \approx 190 \mathrm{~cm}$ are discussed in the light of reconstructions and simulations at $R \approx 203 \mathrm{~cm}$. A top view of the DIII-D tokamak with the active neutral beams and FIDA linesof-sight is shown in figure 1 . The chords intersecting $R \approx 190 \mathrm{~cm}$ and $R \approx 203 \mathrm{~cm}$ are highlighted in blue and red, respectively.

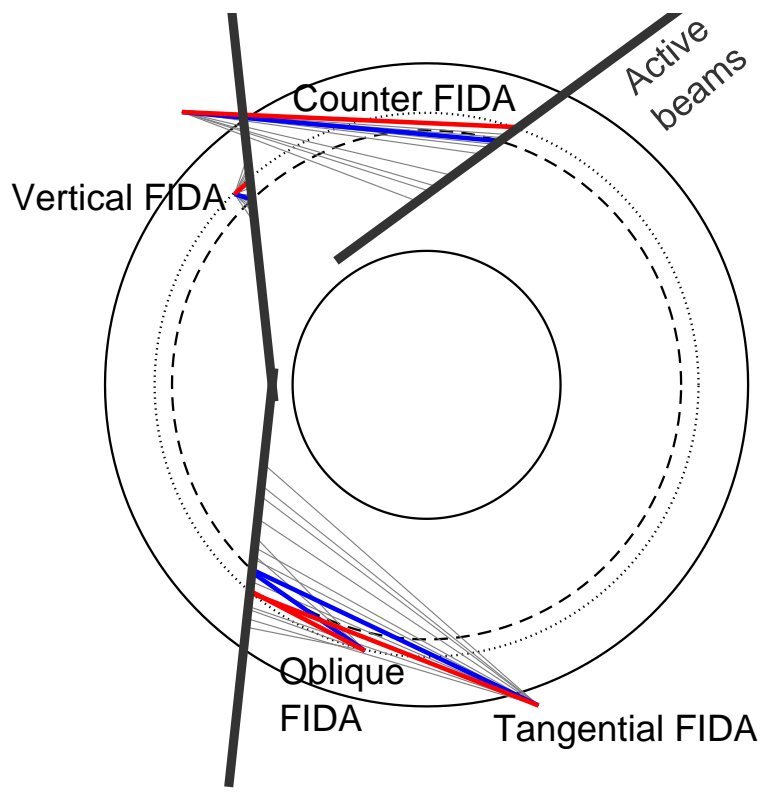

Figure 1. Top view of the four FIDA views looking onto the three active neutral beams in the DIII-D tokamak during discharges \#153071 and \#153072. The highlighted FIDA chords intersecting the beams at $R \approx 190 \mathrm{~cm}$ (dashed circle) and $R \approx 203 \mathrm{~cm}$ (dotted circle) are the ones used for this study.

The measurements are obtained over quasistationary conditions in the time interval $t=2.7-3.7 \mathrm{~s}$. The quasi-stationarity of the discharges is evident from figure 2 that shows the instability spectra for the considered time interval during both discharges (slightly longer than for figure 4 in [20] and figure 1b-c in [21]). Although non-zero, the mode activity during \#153071 is much weaker than during the sequential discharge \#153072. Four distinct types of instabilities are detected in the spectra. These are the ellipticityinduced AEs (EAE, 150-180 kHz), toroidicity-induced AEs (TAE, $90-150 \mathrm{kHz}$ ), additional low-frequency modes $(30-80 \mathrm{kHz})$, and NTMs $(\sim 30 \mathrm{kHz})$. The low-frequency modes are interpreted as AEs in the gaps below the TAE gap, originating from the coupling between Alfvénic and acoustic branches These are likely to be beta-induced AEs (BAEs) 32] that are expected to contribute significantly to the overall fast-ion transport 33,34 . Presently their exact identification is, however, still uncertain.
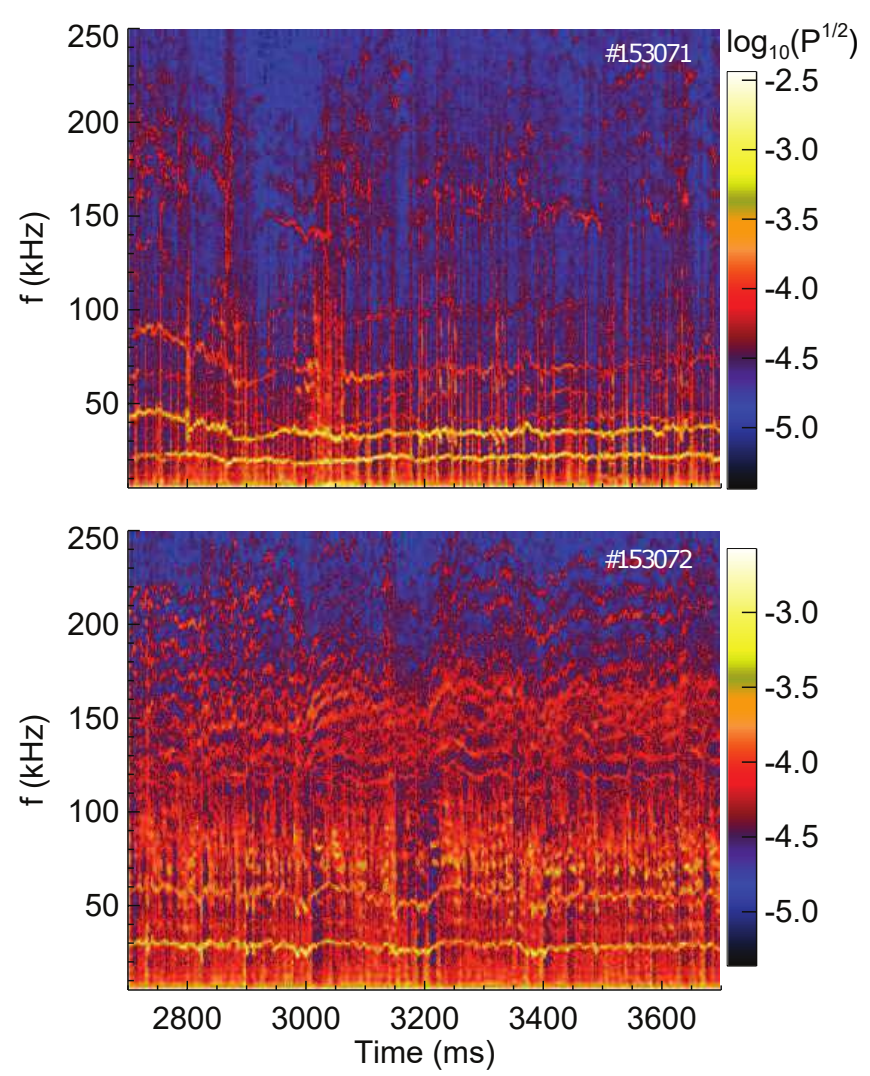

Figure 2. Instability spectra for shot \#153071 (top) and \#153072 (bottom) during the analyzed steady-state portion of the discharges. The spectra are derived from the cross power of radial and vertical CO2 interferometer chords.

The much larger fast-ion transport in discharge \#153072 (strong mode activity) compared to discharge \#153071 (weak mode activity) is clearly seen by considering the fast-ion stored energy and the neutron rate in figure 3 . These are both global quantities that depend upon the volume-averaged fast-ion content. In the figure these quantities are compared with TRANSP/NUBEAM 35] calculations that assume no transport by instabilities. In the low-activity discharge (\#153071), both the fast-ion stored energy and the neutron rate are consistent with the neoclassical TRANSP/NUBEAM predictions within experimental uncertainties. In contrast, in the discharge with strong activity (\#153072), these quantities are $72 \%$ and $61 \%$ of the neoclassical values. When comparing successive discharges, the relative uncertainties are much smaller than the absolute uncertainties (only a 
few percent).
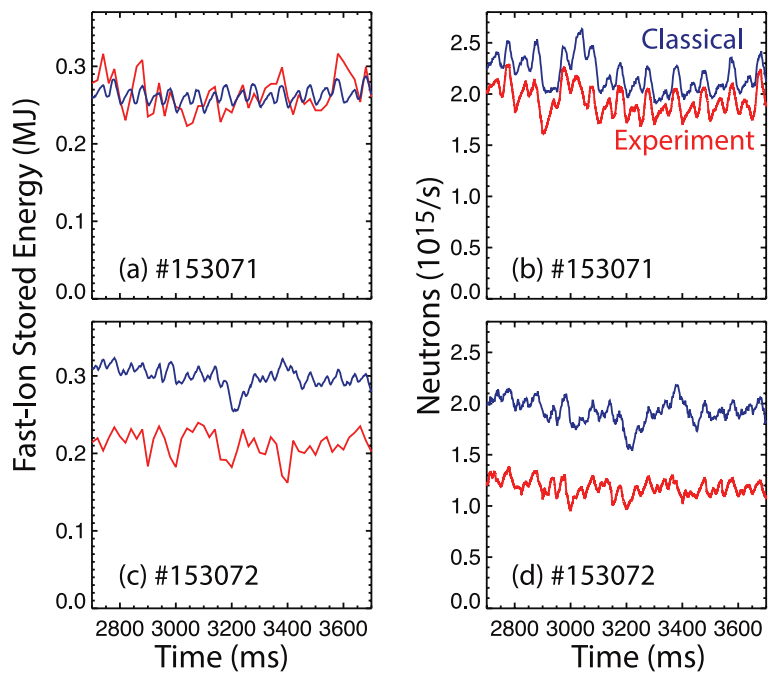

Figure 3. Comparison of measured and calculated global quantities: (a) fast-ion stored energy and (b) neutron rate during discharge \#153071, and (c) fast-ion stored energy and (d) neutron rate during discharge \#153072. The neoclassical predictions neglect all instability-induced transport. The fast-ion stored energy is the difference between the total stored energy and the measured thermal stored energy, with estimated absolute uncertainties of $\sim$ $10 \%$. The absolute uncertainty in the neutron measurement is $\sim 15 \%$.

The neoclassically expected TRANSP/NUBEAM simulations of the fast-ion distributions in the measurement volume at $R \approx 190 \mathrm{~cm}$ are plotted in figure $4 \mathrm{a}$-b for both discharges. These are presented in the commonly used energy $E=\frac{1}{2} m_{D} v^{2}$ and pitch $p=$ $v_{\|} / v$ coordinates where $v_{\|}$is the velocity component along the magnetic field, $v$ is the speed, and $m_{D}$ is the deuterium mass. During the discharges, the fast-ion population is generated mainly by NBI heating with a full injection energy of $80 \mathrm{keV}$ at positive pitches. The discharges are designed to be comparable. However, in the discharge with strong mode activity (\#153072), the beam power is slightly increased relative to discharge $\# 153071$ in order to sustain the high $\beta_{N}$ 20]. This is reflected in increased fast-ion densities at $p \approx 0.5$ (figure 44).

Figure 5 shows the 1D energy spectra of the thermal and fast ion populations in figure 4 obtained by integrating over pitch for $p>0$, where the majority of fast ions are located. The time-averaged local parameters of the thermal distributions are given in table 1. The phase-space density for $E<20 \mathrm{keV}$ greatly exceeds the phase-space density for $E>$ $20 \mathrm{keV}$. In tomographic reconstructions from sparse datasets sensitive to low-energy ions, the high phasespace densities at low energies complicates reliable reconstructions of the dilute high-energy population
Table 1. Time-averaged local parameters with temporal standard deviations of the thermal-ion population at $R \approx$ $190 \mathrm{~cm}$ during discharges \#153071 and \#153072. $T_{i}$ : thermalion temperature, $n_{i}$ : thermal-ion density, $v_{\text {drift }}$ : drift speed related to the toroidal rotation frequency $f_{\text {rot }}$ through $v_{\text {drift }}=$ $2 \pi R f_{\text {rot }}$. Additional time traces and radial profiles for the discharges can be found in 20 .

\begin{tabular}{|l|c|c|}
\hline & $\# 153071$ & $\# 153072$ \\
\hline$T_{i}[\mathrm{keV}]$ & $5.7 \pm 0.2$ & $4.5 \pm 0.2$ \\
\hline$n_{i}\left[\mathrm{~m}^{-3}\right]$ & $(3.3 \pm 0.1) \times 10^{19}$ & $(2.6 \pm 0.1) \times 10^{19}$ \\
\hline$v_{\text {drift }}[\mathrm{m} / \mathrm{s}]$ & $(2.4 \pm 0.2) \times 10^{5}$ & $(2.1 \pm 0.1) \times 10^{5}$ \\
\hline
\end{tabular}

36]. Therefore, FIDA measurements sensitive to ions with energies below $\sim 20 \mathrm{keV}$ are excluded from this study. The exact lower wavelength limit is chosen individually for each view depending on the shapes and sensitivities of the weight functions, in order to retain as much data as possible whilst still avoiding artifacts in the target area caused by high densities at low energies.

The fast-ion information can be extracted from the FIDA spectra at large Doppler-shifts from the unshifted wavelength $\lambda_{0}=656.1 \mathrm{~nm}$. However, the full spectra also contain radiation from neutralized thermal ions, cold edge neutrals, beam neutrals, impurities, and visible bremsstrahlung [26]. Since the bremsstrahlung is fairly constant throughout the relevant wavelength ranges, this can be subtracted from the spectra 37. Parts of the spectra dominated by radiation from the beam neutrals or impurities are omitted in the tomographic inversions of the fast-ion distribution. In addition, in order to avoid saturation from the much stronger D-alpha light from the cold plasma edge, only either the red- or blue-shifted part of each spectrum is measured. For the FIDA diagnostics in the NSTX [38, ASDEX Upgrade 13 and EAST 39 40 tokamaks, the parts of the spectra close to the unshifted D-alpha line is instead notched out allowing measurements in both the red- and blue-shifted ranges.

At ASDEX Upgrade, the lines-of-sight of the five FIDA views intersect the same neutral beam [13]. The beam emission features in the spectra can, therefore, be used to cross-calibrate the signals. This is not directly possible for the DIII-D FIDA system because three different beams are used as neutral donors. Instead, for each view one calibration factor is found from least-squares fits between the measurements and the FIDASIM 41, 42 simulation of the FIDA spectra from the neoclassical TRANSP/NUBEAM distribution during discharge \#153071. This is fair since the neoclassical TRANSP/NUBEAM simulation is expected to be a reasonable model for the true fastion distribution during discharge \#153071 where the measured mode activity is relatively weak, despite the NTM at $\sim 30 \mathrm{~Hz}$, and the neoclassically predicted 
(a) $\mathbf{F}_{\text {TR71 }}$

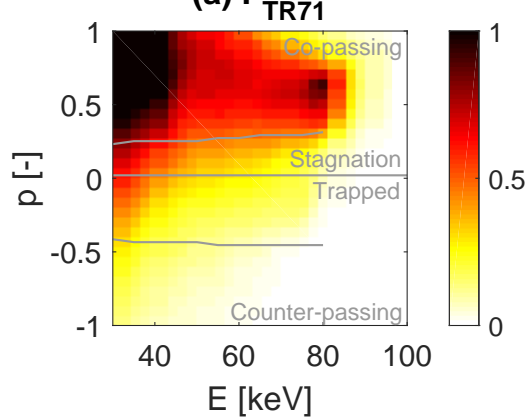

(b) $F_{\text {TR72 }}$

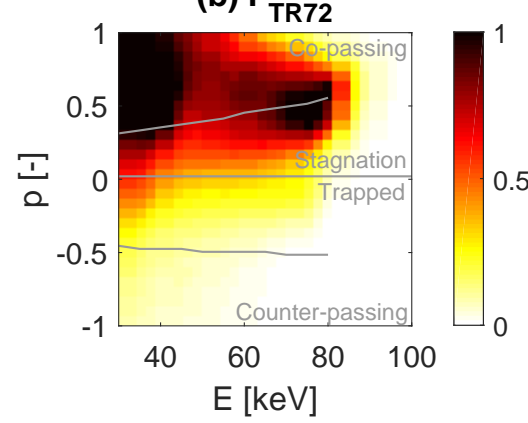

(c) $F_{T R 72}-F_{T R 71}$

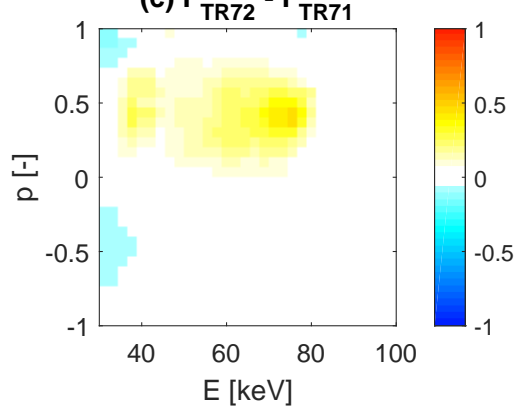

Figure 4. Neoclassical TRANSP/NUBEAM distributions at $R \approx 190 \mathrm{~cm}$ for discharges (a) \#153071 and (b) \#153072 with approximate velocity-space boundaries between different orbit types marked in grey, and (c) pixel-difference between the distributions. The plots are scaled by the same factor. The lower limit of $30 \mathrm{keV}$ corresponds to the energy limit above which the DIII-D FIDA diagnostic allows proper reconstructions from measurements.

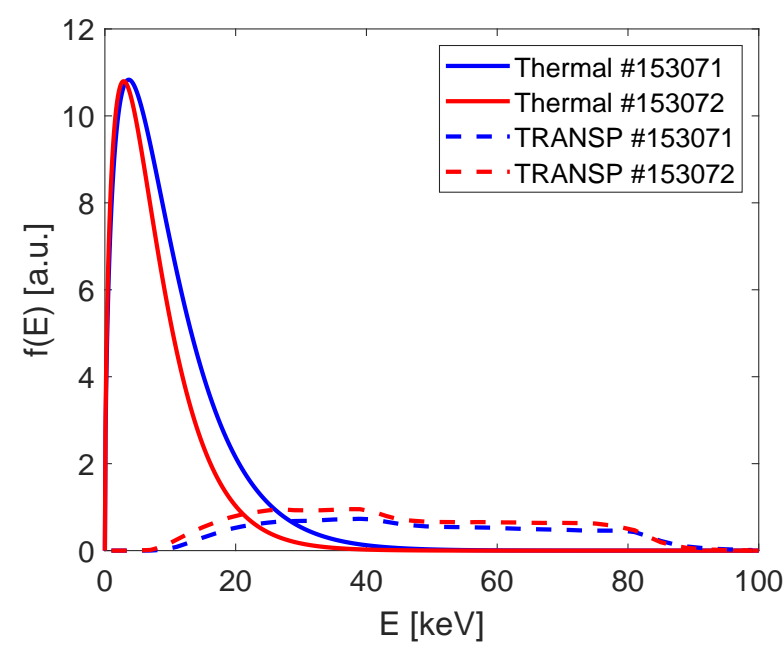

Figure 5. Thermal- and fast-ion energy distributions at $R \approx 190 \mathrm{~cm}$ obtained by integrating over pitch for $p>0$ at the measurement location during discharges \#153071 (blue) and \#153072 (red).

neutron rate is within the uncertainty of the absolutelycalibrated volume-averaged neutron rate (see figure 3). Calibrating the four views in this way is more uncertain and leads to less reliable absolute densities of the reconstructed fast-ion distributions. However, the calibration is not expected to differ for discharges \#153071 and \#153072. Therefore, discharge \#153071 can be used as a calibration and reference shot for discharge \#153072, allowing studies of the changes in velocity space related to the increased mode activity.

The time-averaged calibrated measurements in the experimentally achievable wavelength ranges during discharges \#153071 and \#153072 are shown in figure6 together with the neoclassically expected signals computed with TRANSP/NUBEAM and FIDASIM. The signals have been carefully selected to avoid ELM contamination and NBI-off phases have been applied for background subtraction, as described in [20]. At the smallest Doppler shifts in the measurements, the spectra are affected by the thermal-ion population. The wavelengths ranges in which ions with energies smaller than $\sim 20 \mathrm{keV}$ could cause a signal are marked in grey in the figure 6. The corresponding measurements are not included in the inversions in section 5 in order to enable reliable reconstructions of the low-density high-energy population despite the high densities at low energies.
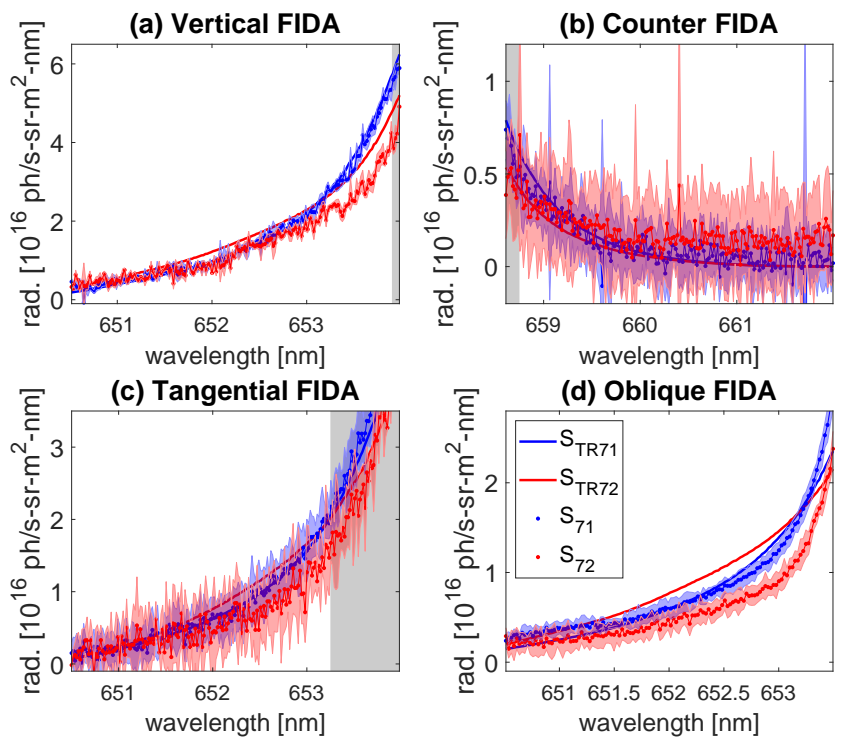

Figure 6. Time-averaged FIDA measurements for each view at $R \approx 190 \mathrm{~cm}$ during discharges \#153071 (weak mode activity, blue) and \#153072 (strong mode activity, red). The measurements are overlaid with the simulated FIDA signals for each diagnostic arising from the TRANSP/NUBEAM fast-ion distributions and thermal-ion populations. The shaded areas enclosing the measurements indicate the measurement uncertainties. 


\section{Tomographic inversion methods}

The signal $s$ relates to the distribution $f$ through known weight functions $w$ 43, 44 by

$s=\int_{-1}^{1} \int_{0}^{\infty} w f d E d p$.

By introducing the vectors $S$ and $F$ that contain the measurements and the distribution, respectively, along with the transfer matrix $W$ containing the weight functions, the forward problem of (1) can be discretized and reformulated in the computationally more convenient matrix form 8

$S=W F$.

We now wish to determine the distribution from the known signal and the known transfer matrix. However, this is an ill-posed problem [15, and in order to obtain a stable solution, the problem has to be regularized. It has previously been shown that the first-order Tikhonov regularization method, which penalizes steep gradients in the solution, together with a non-negativity constraint, provides a good framework for the reconstruction of the central fastion distribution from FIDA measurements in tokamak plasma conditions 14]. Then the solution is given by

$$
\begin{gathered}
F^{*}=\min _{F}\left\|\left(\begin{array}{c}
W \\
\lambda_{1} L_{1}
\end{array}\right) F-\left(\begin{array}{c}
S \\
0
\end{array}\right)\right\|_{2} \\
\text { subject to } F \geq 0
\end{gathered}
$$

where $L_{1}$ is a matrix that produces finite-difference approximations to the gradients with respect to $E$ and $p$ [15], and $\lambda_{1}$ is the regularization strength. In order to account for the signal-to-noise ratios of the individual data points in the least-squares solution of (3), we normalize the measurement and corresponding weight functions by the measurement uncertainty [9].

The solution can be improved at high energies by imposing null-measurements in the reconstruction. These are the parts of the FIDA spectrum where the measurements are below the detection limit of the diagnostic. High densities in the related regions in velocity space are deemed unlikely. Null-measurements have previously been included as a hard constraint using $F\left(E_{0}, p_{0}\right)=0$, where $\left(E_{0}, p_{0}\right)$ is the region in velocity space related to the null-measurements 14, and as an increased penalty on solution norms in the null-measurement velocity-space region 16. Here, we choose the latter method that allows small densities in the null-measurement region and has a smooth transition in the penalty term between the densely populated and the null-measurement regions, reducing boundary effects. The solution is then given by

$$
\begin{gathered}
F^{*}=\min _{F}\left\|\left(\begin{array}{c}
W \\
\lambda_{1} L_{1} \\
\lambda_{0} \xi L_{0}
\end{array}\right) F-\left(\begin{array}{c}
S \\
0 \\
0
\end{array}\right)\right\|_{2} \\
\text { subject to } F \geq 0
\end{gathered}
$$

where $\xi=\xi(E, p)$ is the penalty function in the nullmeasurement region chosen to gradually increase with energy, $L_{0}$ is the identity matrix, and $\lambda_{0}$ determines the strength of the null-measurement penalty term 16. $\lambda_{0}$ is chosen to be strong enough to suppress clearly artificial densities in the null-measurement region without causing significantly increased densities at the boundary into this region.

When reconstructing from a synthetic spectrum where the true solution is known, we determine the regularization strength $\lambda_{1}$ such that the Euclidean norm of the pixel-difference between the reconstruction and the true solution is minimized for $p>0$ and $E>30 \mathrm{keV}$ where the majority of fast ions are located. Different schemes have been suggested for determining the optimal value of $\lambda_{1}$ when reconstructing from measurements [45]. However, none has been shown to consistently provide the best solutions 14,46. For the reconstructions from measurements, we instead choose $\lambda_{1}$ such that spurious features are suppressed without introducing overregularization. From this, we typically find a $\lambda_{1}$ within an order of magnitude from the regularization strengths providing the best reconstructions of the neoclassical TRANSP/NUBEAM simulations from synthetic spectra, where small changes in $\lambda_{1}$ do not drastrically change the shape of the reconstructions.

\section{Sparse velocity-space coverage}

Each spectral measurement originates from neutralized fast ions in a well-defined velocity-space region given by the weight functions. The gross shapes of the FIDA weight functions are determined by the viewing geometry and observed wavelength ranges [43. Summing up all weight functions related to the measurements for each view gives the total velocityspace coverage of that view [16 31. This is illustrated in figure 7 for the four DIII-D FIDA views. The vertical, tangential, and oblique FIDA views are mostly sensitive to the positive-pitch fast-ion population, whereas the counter view is sensitive to the negativepitch population. The strong asymmetries of the summed weight function sensitivities about $p=0$ appear because only one side of each spectrum is available, as is also the case for the FIDA diagnostics in the MAST tokamak 16, 47. Two-sided spectra would provide complete coverage of velocity space in at least two views, as for ASDEX Upgrade [13.

In order to reliably reconstruct the fast-ion velocity distribution from experimental measurements, the target area needs to be covered by more than one view 8, 25, 31. However, as evident from figure 7. only the counter FIDA view is highly sensitive to the negative-pitch range, making proper reconstructions 

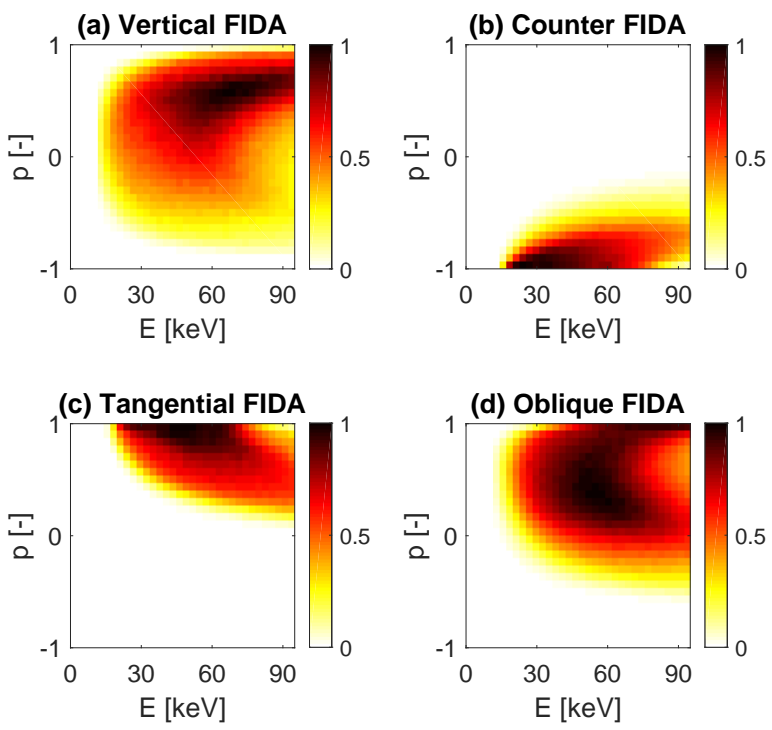

Figure 7. Velocity-space coverage of the weight functions for the highlighted chords of each of the four FIDA views (see figure 1) in the accessible measurement wavelength ranges, i.e. $\lambda_{\text {vert }} \in[650.5: 653.9] \mathrm{nm}, \lambda_{\text {ctr }} \in[658.75:$ 662] $n m, \lambda_{t a n} \in[650.5: 653.25] \mathrm{nm}$, and $\lambda_{o b l} \in[650.5:$ 653.5] $\mathrm{nm}$.

difficult for $p<0$ without including stronger prior information, such as suggested in $[16$. Figure 8 illustrates this problem. Here a test distribution composed of two Gaussian blobs located symmetrically about $p=0$ (figure 8 a) is reconstructed using (3) from a synthetic signal employing the FIDA wavelength grid, noise level, and viewing geometry available during the considered discharges. For positive pitches, the resulting reconstruction (figure 8p) is in good agreement with the true distribution. However, the lack of multiple view coverage for negative pitches causes the density of the negative-pitch Gaussian blob to be smeared out in energy. The poor reconstructions for $p<0$ indicates that we cannot expect credible reconstructions from experimental measurements in this region. Reliable reconstructions can, however, still be obtained for $p>0$.

One way of dealing with this is to introduce a $2 \mathrm{D}$ bin in which ions outside our target area can be deposited. In this approach, we do a standard $2 \mathrm{D}$ reconstruction, but interpret the result as reliable for $p>0$, which is observed by three FIDA views, and unreliable for $p<0$, which is observed by only one FIDA view. Figure 8 illustrates that the DIIID FIDA system allows this approach. Alternatively, information about the behaviour of $f(E, p)$ in either pitch or energy in a restricted part of velocity space can be used to constrain the solution in that region in order to improve the solution in the sparsely diagnosed region. The TRANSP/NUBEAM simulation suggests that the velocity distribution is monotonic in a significant part of velocity space. In particular, the phase-space density increases monotonically with increasing pitch from $p=-1$ to $p=0.5$ (see figure 4 b). This can be included as a constraint in (3) and (4) such that the problem becomes

$$
\begin{gathered}
F^{*}=\min _{F}\left\|\left(\begin{array}{c}
W \\
\lambda_{1} L_{1} \\
\lambda_{0} \xi L_{0}
\end{array}\right) F-\left(\begin{array}{c}
S \\
0 \\
0
\end{array}\right)\right\|_{2} \\
\text { subject to }\left\{\begin{array}{l}
F \geq 0 \\
L_{p}^{-} F \geq 0
\end{array}\right.
\end{gathered}
$$

where $L_{p}^{-}$is a matrix that produces finite-difference approximations to the gradient with respect to pitch in the region $p<0$. Here, we have chosen $p=0$ as the limit below which the monotonicity constraint is applied instead of $p=0.5$ in order to minimize the constraint on the solution. A similar prior assuming monotonicity in energy instead of pitch might also prove useful in reconstructions of the slowingdown distribution of fusion-born alpha-particles that in the simplest picture is isotropic and monotonically decreasing with energy [31].

Prior information can also be used to reduce the problem size and altogether circumvent reconstructing the distribution in the velocity-space regions that are not sufficiently diagnosed. Depending on the imposed prior information, this method might provide a better reconstruction in the well-diagnosed regions than the full 2D reconstruction, but more importantly allows reconstruction in only the well-diagnosed regions. Additionally, the choice of prior might also indicate the structure of the distribution in the sparsely diagnosed region, since strong deviations of the prior from the true distribution will also affect the reconstruction in the well-diagnosed region given that the dataset includes measurements sensitive to the region affected by the prior. Hence, reconstructing in only a restricted part of velocity space can effectively be a combination of forward modelling and reconstruction which might also prove to be a strong tool in further studies on the fast-ion distribution from datasets with sparse velocityspace coverage.

In this study, we propose to reconstruct the distribution for only $p>0$, where the majority of the fast-ion population is distributed and impose a prior on the negative pitch range. Since some of the weight functions are sensitive to both the positive- and negative-pitch regions, this requires a reformulation of the problem. In the following section, we suggest two different priors to do so.

\subsection{Restricted velocity-space inversion methods}

Consider the integral formulation of the forward problem in (1). The integral over pitch can be split 
(a) $F_{T}$

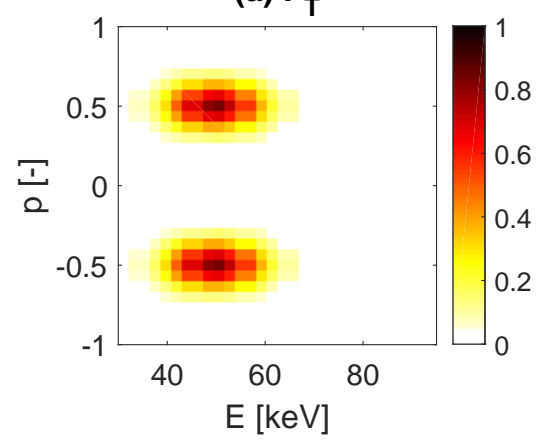

(b) $F^{*}$

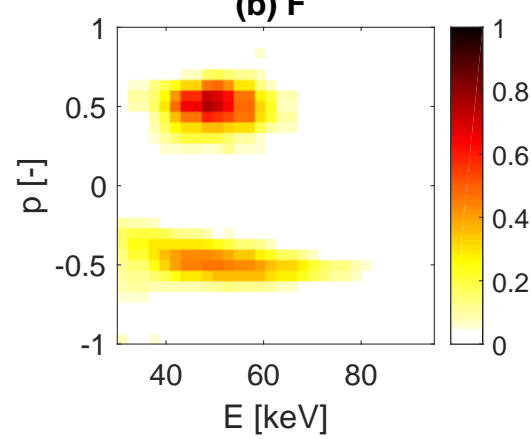

(c) $F^{*}-F_{T}$

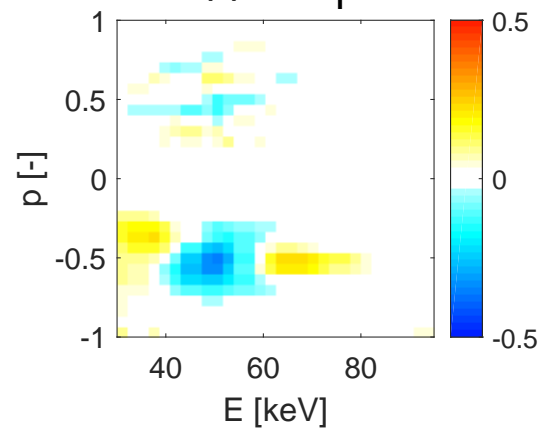

Figure 8. (a) Test distribution, (b) tomographic reconstruction of (a), and (c) pixel-difference between the reconstruction and the test distribution.

into two integrals:

$s=\int_{-1}^{0} \int_{0}^{\infty} w^{-} f^{-} d E d p+\int_{0}^{1} \int_{0}^{\infty} w^{+} f^{+} d E d p$

where the superscripts indicate the positive $(+)$ and negative $(-)$ pitch regions. Note that the pitch boundary can be arbitrarily chosen and is not restricted to be $p=0$. Here we choose $p=0$ as our boundary and stick to this notation. We now introduce the signals $s^{+}$and $s^{-}$originating from ions with positive and negative pitches, respectively, i.e.

$s^{-}=\int_{-1}^{0} \int_{0}^{\infty} w^{-} f^{-} d E d p$

and

$s^{+}=\int_{0}^{1} \int_{0}^{\infty} w^{+} f^{+} d E d p$

such that $s=s^{-}+s^{+}$.

We seek $f^{+}$, but know only $s$ and not $s^{+}$. If $s^{-}=0$, a proper solution can be found for the matrix problem $S=W^{+} F$. However, if $s^{-} \neq 0$, the assumption that $s^{-}=0$ will cause a distortion of the reconstruction at positive pitches, likely with increased densities in some regions. One way of overcoming the lack of information about $s^{-}$is to use a known distribution from a simulation in the negative-pitch region as prior information. That is, we introduce the known quantity

$s_{\text {sim }}^{-}=\int_{-1}^{0} \int_{0}^{\infty} w^{-} f_{\text {sim }}^{-} d E d p$

and search for the solution to the problem

$s-s_{\text {sim }}^{-}=\int_{0}^{1} \int_{0}^{\infty} w^{+} f^{+} d E d p$.

This results in a good solution if the simulation is exact for $p<0$. However if not exact, this prior will impose untrue information on the problem possibly disturbing the reconstruction for $p>0$.
An alternative way of reconstructing the distribution for only positive pitches is to introduce a 1D energy-resolved bin in which the fast ions with negative pitches can be deposited. This can be done by assuming that $f^{-}$is separable into an arbitrary energy distribution and a known pitch distribution, i.e. $f^{-}(E, p)=f_{1 D}^{-}(E) f_{1 D}^{-}(p)$. Here we assume $f^{-}$to be isotropic, i.e. it does not depend on pitch. Then the signal originating from ions with negative pitches is

$s^{-}=\int_{0}^{\infty} f^{-} \int_{-1}^{0} w^{-} d p d E$.

Now we introduce

$w^{-}(E)=\int_{-1}^{0} w^{-}(E, p) d p$

such that

$s^{-}=\int_{0}^{\infty} f^{-} w^{-}(E) d E$.

In matrix form this is

$S^{-}=W_{1 D}^{-} F_{1 D}^{-}$.

We now seek the solution to

$S=S^{+}+S^{-}=\left[\begin{array}{ll}W^{+} & W_{1 D}^{-}\end{array}\right]\left[\begin{array}{l}F^{+} \\ F_{1 D}^{-}\end{array}\right]$.

A distribution with a pitch-independent $F^{-}$is well-reconstructed with this method. However, if the distribution is not isotropic for $p<0$, the isotropy assumption might introduce systematic biases in the reconstruction. Hence, this method works best for problems with isotropic distributions or low densities in the negative-pitch region.

\section{Reconstructing the fast-ion distribution at DIII-D}

In order to reconstruct a velocity distribution with the weight function coverage provided by the FIDA diagnostics in the DIII-D tokamak using these 
methods, the negative-pitch part of the distribution must be low-density, pitch-independent, monotonic, or known. These assumptions are justified to some extent for the neoclassically expected distributions during the considered DIII-D discharges: i.e. the main part of the neoclassically expected fast-ion population has positive pitches, and for $p<0$, the low-density distribution is monotonically increasing with pitch from $p=-1$ to $p=0$. This is clearly reflected in figure $4 \mathrm{a}-\mathrm{b}$.

Here, we evaluate how the 2D bin, the monotonicity constraint and the restricted velocity-space inversion methods affect the reconstruction of a realistic distribution. For this purpose we invert synthetic FIDA signals based on the neoclassical TRANSP/NUBEAM simulation for discharge \#153071. As for the synthetic signal used for the reconstruction in figure 8, we interpolate the synthetic signal onto the experimentally accessible wavelength grid and add noise determined by the measurement uncertainty. In all reconstructions, we constrain the solution to be non-negative, and we include the experimental null-measurements as prior information such that the solutions are determined from (4) and (5). A set of resulting reconstructions are shown in the top panel of figure 9. The lower panel gives the pixel-differences between the reconstructions and the true solution.

Figure $9 \mathrm{a}$ gives the full reconstruction with the $2 \mathrm{D}$ bin for $p<0$. The distribution is wellreconstructed for positive pitches although the fullenergy injection peak is shifted to lower energies. In other fast-ion velocity-space tomography studies, resolving the injection peak position and density accurately without including extra prior information about the beam location has also proven challenging 14. 15]. In the 2D bin, especially around $p=-0.7$, the distribution has artefacts. Here, the reconstructed distribution is smeared out towards higher energies in a similar fashion as in figure 8 $8 \mathrm{p}$. When including the monotonicity constraint for negative pitches, the spurious protrusion at $p=-0.7$ in figure $9 \mathrm{a}$ is not allowed and hence does not appear, whereas the distribution at positive pitches does not change significantly. For positive pitches, both restricted velocity-space inversion methods give reconstructions similar to the true solution. The 1D bin method (figure 9c) distributes the ions slightly differently than the $2 \mathrm{D}$ bin and monotonicity reconstructions, however, without drastically increasing the error. Including the simulated signal originating from the negative-pitch region of the distribution as prior results in the best reconstruction (figure $9 \mathrm{~d}$ ).

In order to evaluate the reproducibility of these trends, the neoclassical TRANSP/NUBEAM distribution for \#153071 has been reconstructed from 100 synthetic spectra with different noise realizations using the same noise level and diagnostic coverage. The results are presented in table 2 as the average norm of the pixel-differences between the reconstructions and the true solution, i.e.

$\epsilon^{*}=\frac{\left\|F_{T R 71}^{*}-F_{T R 71}\right\|_{2}}{\left\|F_{T R 71}\right\|_{2}}$

and as the average fraction of the reconstructed density to the density of the true solution in various regions. The results are in good agreement with the conclusions reached from figure 9 For negative pitches, the 2D bin and monotonicity-constrained reconstructions suffer from displaced and increased densities. Contrarily, for positive pitches and energies above $30 \mathrm{keV}$, the density is well-reconstructed using all methods, and the norms of the pixel-differences are within $\sim 20 \%$ of the norm of the true solution. Close to the beam injection peak, the norms of the pixel-differences are increased by a factor of 2 relative to the true solution for all but the 1D bin reconstruction. Here, especially the methods reconstructing over all pitches struggle to accurately reconstruct the precise location and density of the injection peak, illustrating that in some cases, the restricted velocity-space methods are superior to the reconstructions for all pitches. We also note that reconstructing for only positive pitches are three to four times faster than full reconstructions which is useful when speed is important.

For $E>30 \mathrm{keV}$ and positive pitches, the reconstruction method employing the simulation as a prior for negative pitches generally provides the reconstruction with the smallest norm of the pixel-difference. Note, however, that whereas the reconstruction using the TRANSP/NUBEAM simulation in $p<0$ as prior information results in good reconstructions from synthetic signals, this might not be the case when inverting the experimentally measured signals. The good agreement between the simulated spectra and the measurements during discharge \#153071 (figure 6) does, however, indicate that $s_{\text {sim }}^{-}$might be a good prior for this case, whereas this is questionable for discharge \#153072.

\subsection{Fast-ion distributions during weak and strong mode activity}

Figure 10 1 -d present the reconstructions from FIDA measurements at $R \approx 190 \mathrm{~cm}$ during discharge \#153071. The positive-pitch range of all reconstructions are comparable in density and overall shape to the NBI fast-ion distribution predicted by the TRANSP/NUBEAM code. For all methods, the injection peak at full energy, that is easily observed in the TRANSP/NUBEAM distribution at $E \approx 80 \mathrm{keV}$, peaks at a lower energy, as was also the case for the reconstructions from synthetic signals. The good agree- 

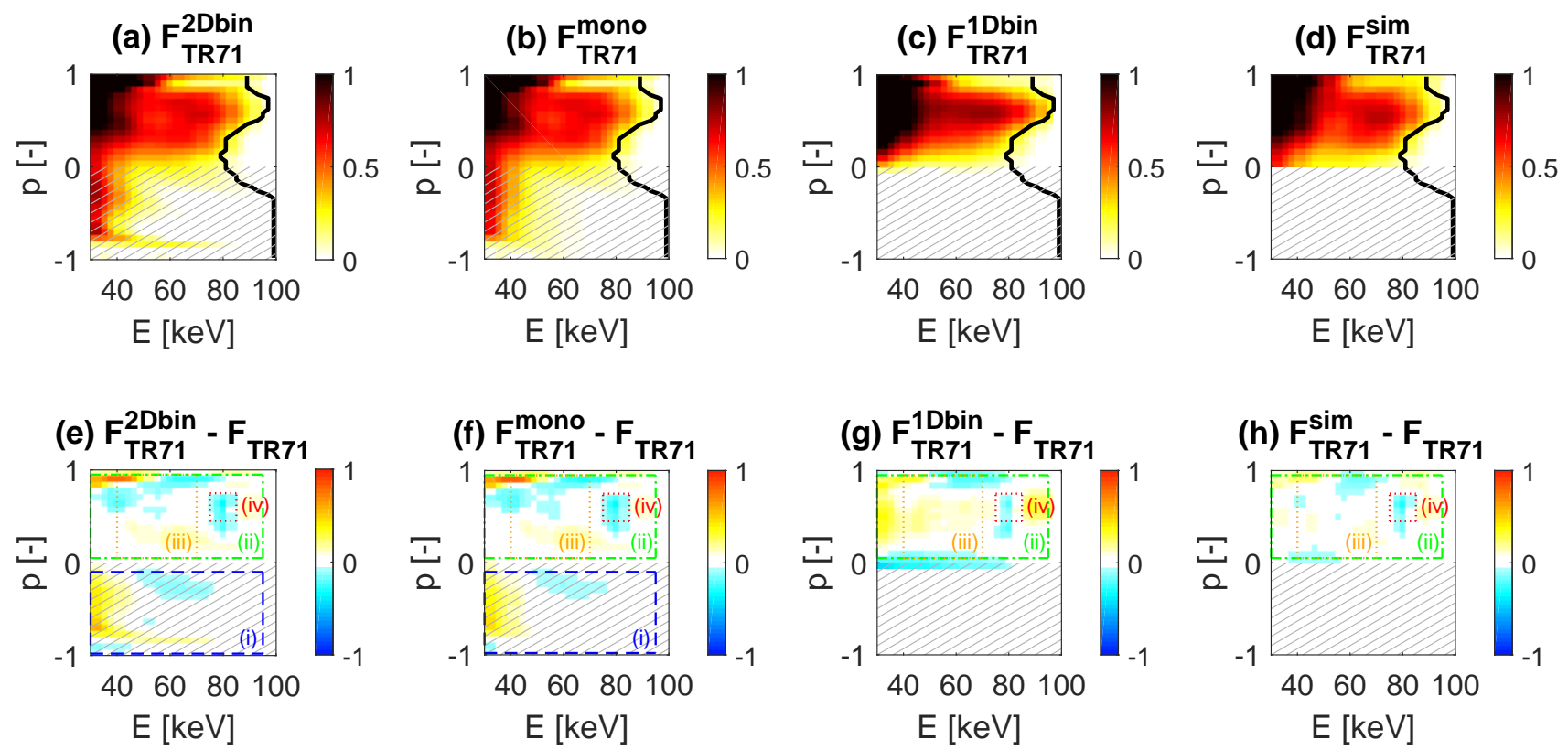

Figure 9. (a-d) Reconstructions of the neoclassical TRANSP/NUBEAM distribution at $R \approx 190$ cm for discharge \#153071 (figure 4a) from a synthetic signal employing DIII-D FIDA diagnostics conditions. The superscripts indicate the used inversion method, i.e. (a) $S=S_{\text {full }}$ and $W=W_{\text {full }}$, (b) $S=S_{\text {full }}$ and $W=W_{\text {full }}$ conditioned $L_{p}^{-} F \geq 0$, (c) $S=S_{f u l l}$ and $W=\left[W^{+} W_{1 D}^{-}\right]$, and (d) $S=S_{\text {full }}-S_{\text {sim }}^{-}$and $W=W^{+}$. The black line is the lower energy limit of the nullmeasurement region. (e-h) Pixel-differences between each reconstruction and the true solution. The regions enclosed by coloured dashed and dotted lines are evaluated in table 2. All panels are scaled by the same factor as in figure 4.

Table 2. Average computing time relative to the $2 \mathrm{D}$ bin reconstruction, and relative norms of the pixel-differences $\epsilon^{*}$ and densities $n^{*} / n_{\text {true }}$ between reconstructions from 100 synthetic spectra and the true solution. This is done for the four reconstruction methods in the different regions marked in figure 9p-h: (i) $E>30 \mathrm{keV}$ and $p<-0.1$, (ii) $E>30 \mathrm{keV}$ and $0.05<p<0.95$, (iii) $40 \mathrm{keV}$ $<E<70 \mathrm{keV}$ and $0.05<p<0.95$, and (iv) $75 \mathrm{keV}<E<85 \mathrm{keV}$ and $0.45<p<0.75$.

\begin{tabular}{l|c|c|c|c|c}
\hline \multicolumn{2}{l|}{} & 2 Dbin & Monotonicity & 1Dbin & Simulation \\
\hline \multirow{2}{l}{ Computing time } & 1.0 & 3.7 & 0.26 & 0.32 \\
\hline \multirow{2}{*}{ (ii) Positive pitches } & $\epsilon^{*}$ & 0.88 & 0.84 & N/A & N/A \\
& $n^{*} / n_{\text {true }}$ & 1.68 & 1.62 & N/A & N/A \\
\hline \multirow{2}{*}{ (iii) Central energies } & $n^{*} / n_{\text {true }}$ & 0.18 & 0.19 & 0.18 & 0.10 \\
& $\epsilon^{*}$ & 0.17 & 0.99 & 1.1 & 1.0 \\
\hline \multirow{2}{*}{ (iv) Injection peak } & $n^{*} / n_{\text {true }}$ & 1.1 & 0.18 & 0.15 & 0.09 \\
& $\epsilon^{*}$ & 0.38 & 0.38 & 0.16 & 0.20 \\
\hline
\end{tabular}

ment between the simulated and reconstructed distributions for the discharge with expected close-to neoclassical transport serves as a validation of our ability to reliably reconstruct the fast-ion distribution for $p>0$ from FIDA measurements. Thus, we can use discharge \#153071 as a reference and calibration shot to study the effect of the increased mode activity on the fast-ion velocity distribution in discharge \#153072.

Apart from the presence of stronger mode activity, discharge \#153072 is designed to be comparable to discharge \#153071 20]. Even so, the reconstructions of the fast-ion velocity distribution at $R \approx 190 \mathrm{~cm}$ during \#153072 (figure 10-h) differ significantly from the corresponding reconstructions for discharge \#153071. This is in part explained by the slightly larger beam power during the discharge with strong mode activity. Figure 10-1 give the pixel-differences between the reconstructions from measurements obtained during the two discharges. Here the slight density increase near the beam injection peak is notable using all inversion methods. However, more remarkable is the substantial decrease in densities throughout most of the positive-pitch region during discharge \#153072 compared to \#153071 observed in all panels. This is not predicted by the neoclassical TRANSP/NUBEAM simulation (figure 4k) and does not appear in 

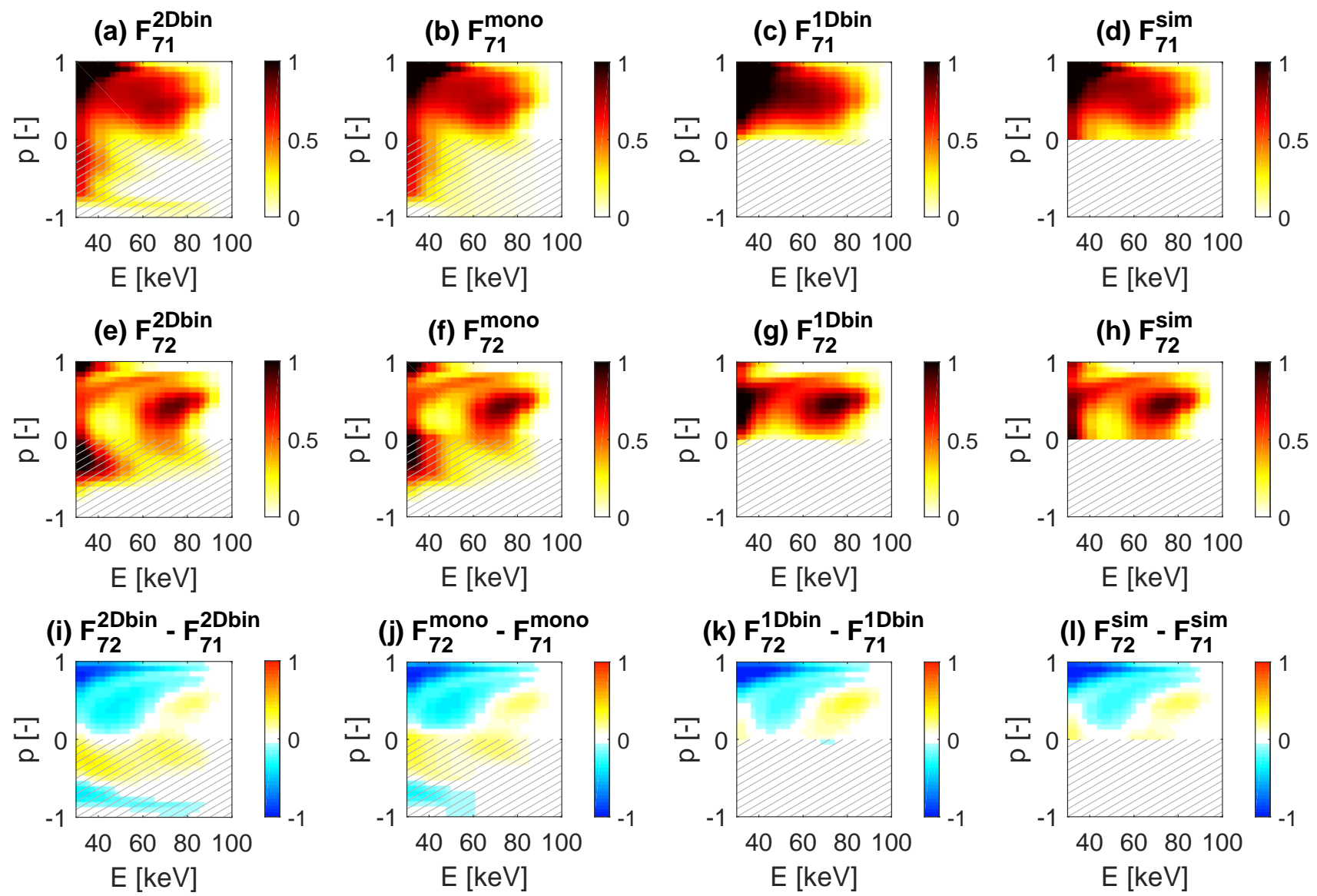

Figure 10. Reconstructions of the fast-ion velocity distribution at $R \approx 190 \mathrm{~cm}$ from measurements obtained during discharges ( $a$-d) \#153071 and (e-h) \#153072, and (i-l) pixel-differences between the reconstructions for discharges \#153071 and \#153072. The notation and scaling factor are the same as in figure 4 and 9.

reconstructions based on synthetic signals from the neoclassical distributions (figure $11 \mathrm{p}$ ). We therefore attribute the observed fast-ion transport to the increased mode activity.
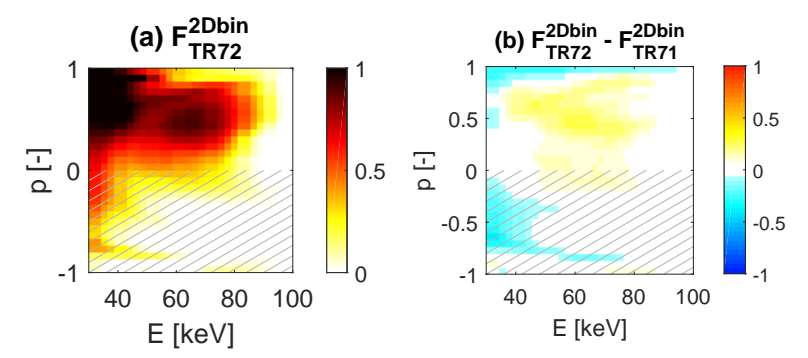

Figure 11. (a) 2Dbin reconstruction of a synthetic signal based on the neoclassical TRANSP/NUBEAM simulation at $R \approx 190 \mathrm{~cm}$ for discharge \#153072, and (b) pixeldifference between the 2Dbin reconstructions from synthetic signals for discharges \#153071 and \#153072. The scaling is the same as in figure 4 .

The strong fast-ion density decrease in discharge \#153072 relative to discharge \#153071 is also observed in reconstructions from FIDA measurements farther out at $R \approx 203 \mathrm{~cm}$, as evident from figure 12 . Here, we present only the distributions reconstructed using the 2Dbin method for the sake of brevity. All four reconstruction methods give similar characteristics at $R \approx 203 \mathrm{~cm}$ as also observed for the comparison at $R \approx 190 \mathrm{~cm}$. As for the local distribution at $R \approx 190 \mathrm{~cm}$, the positive-pitch population at $R \approx 203 \mathrm{~cm}$ experiences large density decreases compared to discharge \#153071 everywhere except for at the beam injection location, where also the neoclassical TRANSP/NUBEAM distributions predict a density increase. Again, the large density decrease is attributed to the increased mode activity.

\section{Kick model results during strong activity}

The kick model module of the TRANSP simulation code 48 provides a way of modelling the effect of the observed AEs and other instabilities on the fast-ion distribution 24]. In order to assess the fast-ion transport caused by the instabilities in the 

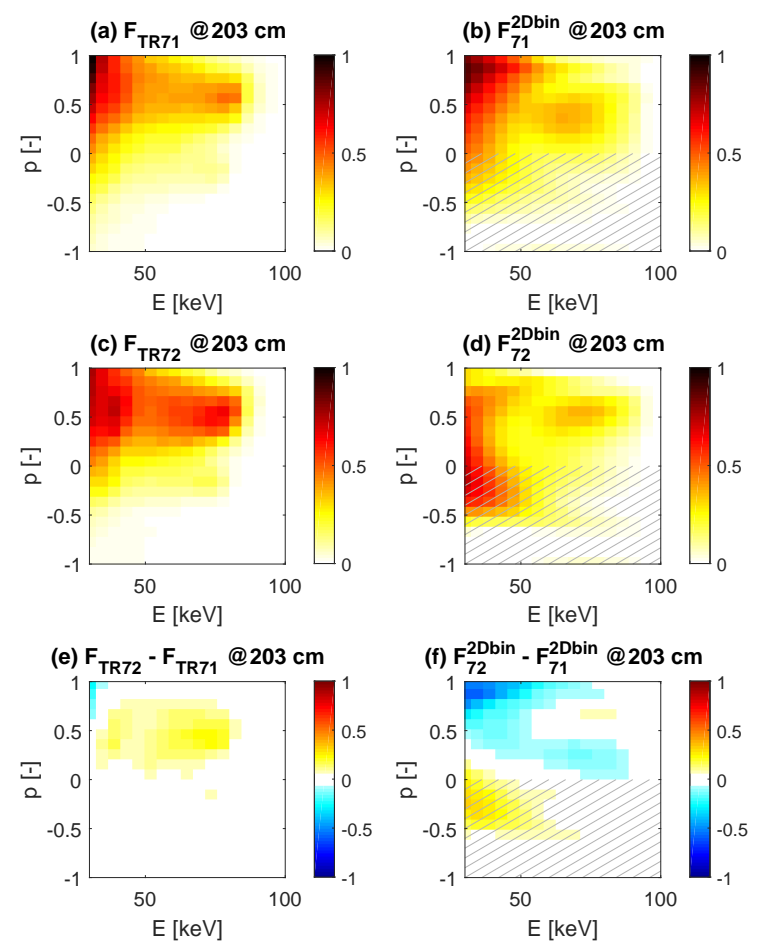

Figure 12. Neoclassical TRANSP/NUBEAM distributions and 2Dbin reconstructions from measurements at $R \approx$ $203 \mathrm{~cm}$ during discharge ( $a-b)$ \#153071 and (c-d) \#153072, and pixel-differences between the distributions (e) as expected by TRANSP/NUBEAM and (f) from reconstructions. The notation and scaling factor are the same as in figure 4 and 11.

kick model, enhanced transport by instabilities is modelled by phase-space resolved transport probability matrices for fast ions in the Monte Carlo NUBEAM module of TRANSP. The matrices are precomputed via particle following codes such as ORBIT [49. Energy and canonical toroidal angular momentum for a sample particle population are evolved in ORBIT in the presence of instabilities, and their changes (or kicks) are recorded as a function of fast-ion phasespace variables (energy $E$, canonical toroidal angular momentum $P_{\zeta}$, and magnetic moment $\mu$ ) to build the transport matrices.

For the TAEs and EAEs, the radial mode structures used as input for ORBIT to compute the kick transport matrices are evaluated by the simulation code NOVA-K [50,51, whereas the NTM mode structures are approximated by an analytical expression [52 based on the mode helicity inferred from Mirnov coil arrays located at the low-field-side vessel wall. Based on the q-profile used in the TRANSP modeling with $q_{m i n} \gtrsim 2$, a $(m, n)=$ $(2,1)$ spectrum is used to simulate the NTM. Note that this is different from the $(3,2)$ identification reported in 20], which appears inconsistent with the $q_{\text {min }} \gtrsim 2$ condition. The radial mode structure of the low-frequency AEs is also introduced in ORBIT through analytical expressions, using the toroidal mode number, mode frequency and radial mode localization from the available experimental data. The radial mode structure $\left(\alpha_{n, m}(\Psi)\right)$ coefficients used as input to ORBIT 49 are assumed to be simple Gaussians with full-width at half-maximum of $\sim 0.25$ in terms of normalized minor radius. Consistent with the ORBIT code implementation, it is assumed that the net radial fast-ion transport is primarily caused by the shear (radial) component of the perturbation. The compressional component is neglected, which introduces an additional arbitrariness in the interpretation of the amplitude of the lowfrequency AEs.

In the TRANSP + kick model simulations, an additional input is used to scale the magnitude of the kicks from the transport probability for each mode as time evolves. This is done in order to mimic possible changes in mode amplitude versus time. For modes such as TAEs and EAEs, for which damping rates can be computed through NOVA-K, time-dependent amplitudes are set so that the mode drive, computed from NUBEAM outputs, equals the damping rate [24]. This procedure is not possible for the NTM and lowfrequency AEs, for which damping rates and other driving terms (for the NTM) are not sufficiently well known. Therefore, their time-averaged amplitudes are adjusted to match the measured neutron rate. Note, however, that since the neutron rate is a global quantity, different combinations of mode amplitudes can lead to the same neutron rate. Hence, adjusting the mode amplitudes to match the measured neutron rate does not guarantee a unique solution for the mode amplitudes. Furthermore, given the uncertainties in the properties of the low-frequency modes (e.g. their radial structure and damping rate), further corrections to account for the possible intermittency in the lowfrequency AE amplitude versus time are not considered in this work.

Radial profiles of the perturbations are shown in figure 13 as a function of the major radius on the midplane. The figure shows that the NTM is localized inside mid-radius, whereas the TAEs and EAEs are mostly localized in the outer plasma region. The lowfrequency AEs have a broader structure, which spans most of the minor radius including regions close to the magnetic axis. The average amplitudes of the different modes used in the kick simulations, given in terms of the relative perturbation of the radial magnetic field component normalized to the total magnetic field $\delta B_{r} / B$, are summarized in table 3 .

The kick model solutions for $R \approx 190 \mathrm{~cm}$ 

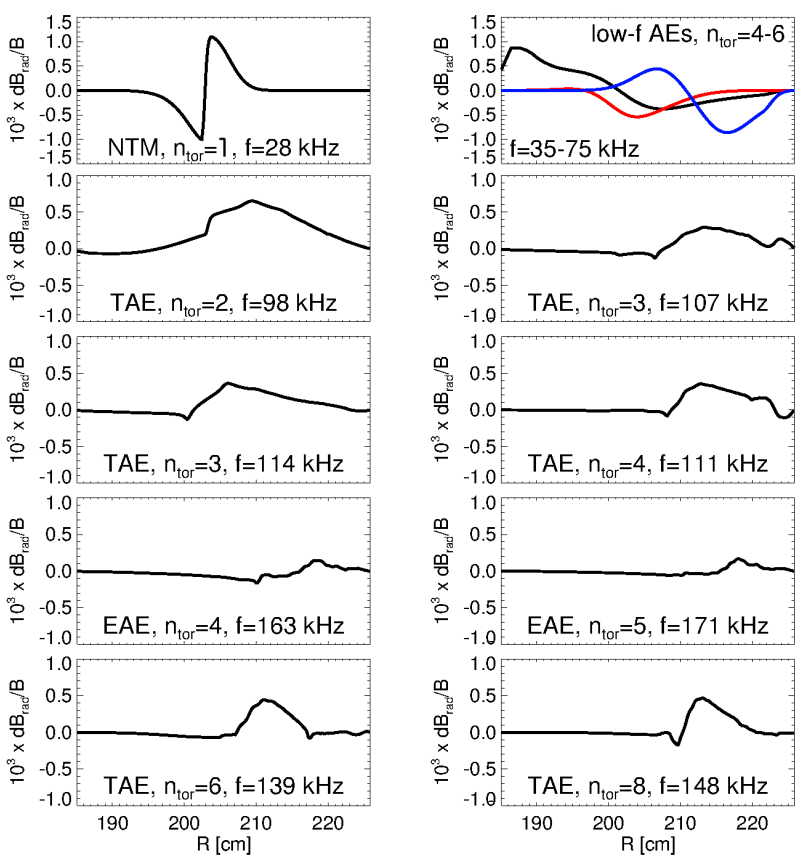

Figure 13. Radial structure of the relative perturbation of the radial magnetic field for the instabilities included in the TRANSP + kick model simulations. Shown here are the average amplitudes used in the kick model simulations, cf. table 3. For the low-frequency AEs, the black, blue and red lines correspond to $n=4, n=5$ and $n=6$, respectively.

Table 3. Magnetic perturbation amplitudes $\left(\delta B_{r} / B\right)$ used for the TRANSP + kick model simulations.

\begin{tabular}{|l|c|c|}
\hline & $\mathrm{f}[\mathrm{kHz}]$ & $\delta B_{r} / B$ \\
\hline TAEs & $90-150$ & $3-7 \times 10^{-4}$ \\
\hline EAEs & $150-180$ & $1-3 \times 10^{-4}$ \\
\hline low-f AEs & $30-80$ & $0.5-1 \times 10^{-3}$ \\
\hline NTM & $\approx 30$ & $1-2 \times 10^{-3}$ \\
\hline
\end{tabular}

and $R \approx 203 \mathrm{~cm}$ using the mode amplitudes given in table 3 are shown in figure $15 \mathrm{a}$-b. In the simulations, we average over the restricted time interval $t=2.5-3.3 \mathrm{~s}$ to save computational time. Due to the quasi-stationary conditions in discharge \#153072 (see the spectrogram in figure 2 and the measured neutron rate in figure 14), this yields similar conditions as observed by the FIDA diagnostics at $t=2.7-3.7 \mathrm{~s}$. Figure 15 -f give the pixeldifferences between the kick model distributions and the neoclassical TRANSP/NUBEAM distribution for discharge \#153071 at the same location. The density decrease caused by the mode activity significantly exceeds the one observed in the reconstructions from measurements at both $R \approx 190 \mathrm{~cm}$ and $R \approx 203 \mathrm{~cm}$. However, recall that the absolute values of kick 1a is based on matching the measured neutron rate, whilst the absolute values of the reconstructions are

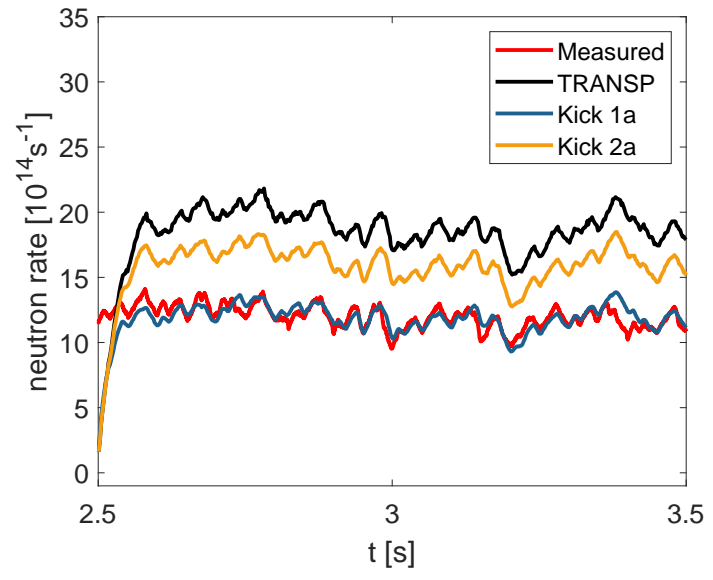

Figure 14. Measured and simulated neutron rates during discharge \#153072 (strong mode activity) from both the neoclassical simulation (TRANSP) and kick model simulations (table 4).

affected by the chosen calibration factors. Owing to the uncertainties in the mode amplitudes and reconstructed densities, we repeat the kick simulation with reduced amplitudes of all modes by $70 \%$ relative to the tabulated values. By doing so, we obtain good agreements between the reconstructions from measurements and the kick model simulation at both $R \approx 190 \mathrm{~cm}$ and $R \approx 203 \mathrm{~cm}$. That is, both the reconstructions from measurements and the kick model detect a decrease in the fast-ion density for almost all positive pitches, except at the beam injection location around $E \approx 80 \mathrm{keV}$ and $p \approx 0.5$.

The computed neutron rates for the two kick model solutions are given in figure 14 . From these it is evident that only the kick 1a solution match the measured neutron rate within the experimental uncertainties as prescribed by the choice of low-frequency mode amplitudes in the simulation. Reducing all mode amplitudes in kick $2 \mathrm{a}$ increases the global confinement and hence the neutron rate well above the measurements. Neither kick 1a nor kick 2a, therefore, give a complete quantitative picture of the fast-ion transport, and the true solution might lie somewhere in-between. Furthermore, due to the uncertainty on the absolute values in the calibration of the FIDA spectra used for the reconstructions, a full quantitative assessment and comparison between reconstructions from measurements and kick model simulations are left for a future study.

Instead, in order to assess the sensitivity of the simulation results and get insight into the effect of the mode activity on the local fast-ion velocity distribution, we scan the amplitudes of the lowfrequency and high-frequency modes, seperately, as summarized in table 4. The amplitudes given in table 3 are set as the reference. Kick 1a-1c scan the low- 

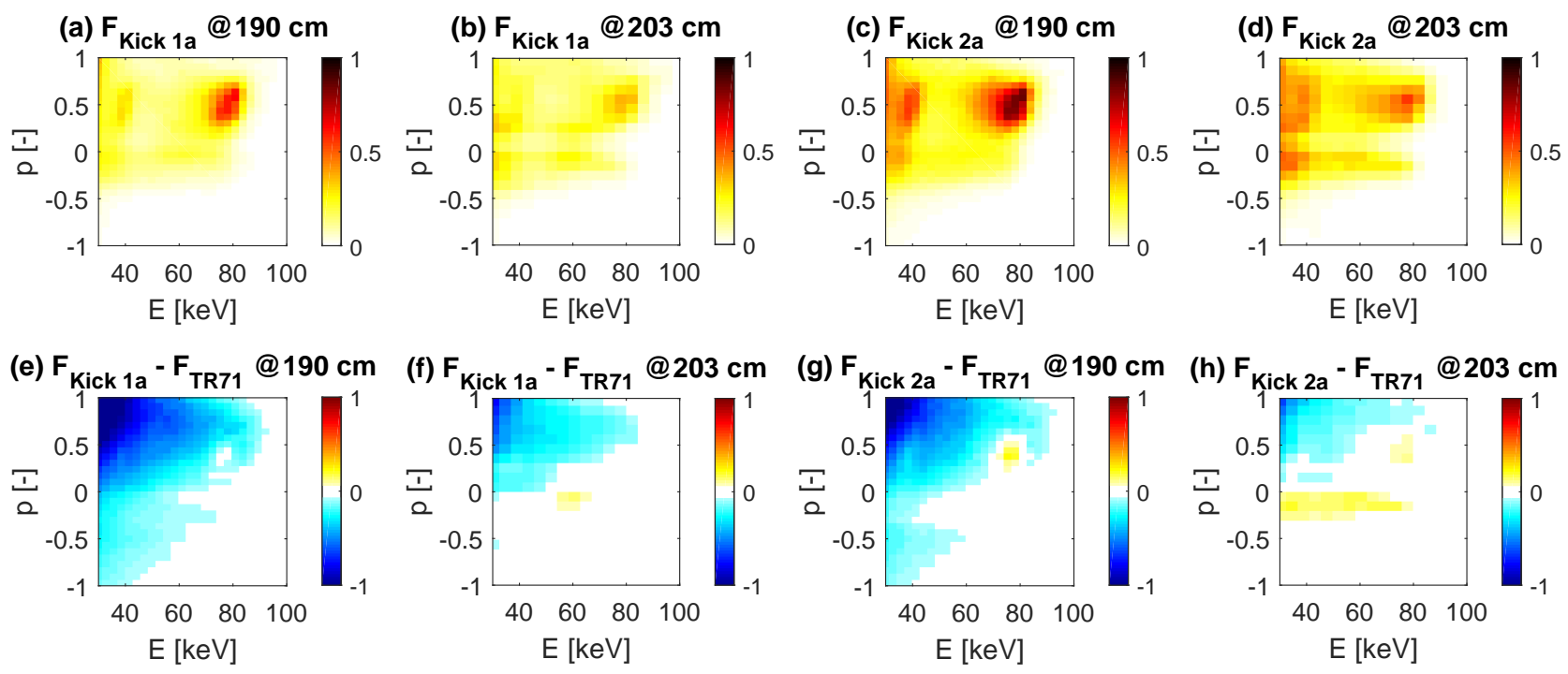

Figure 15. Kick model solutions at $R \approx 190 \mathrm{~cm}$ and $R \approx 203 \mathrm{~cm}$ for discharge \#153072 using (a-b) mode amplitudes given in table 3 (Kick 1a), and (c-d) reducing the mode amplitudes by $70 \%$ (Kick 2a). The lower row gives the pixel-differences between the kick model distributions in the upper row and the corresponding neoclassical distribution for discharge \#153071. The notation and scaling factor are the same as in figure 4

frequency mode amplitude, whereas kick 2a-2c scan the high-frequency mode amplitudes. As extreme cases, kick 1d includes only low-frequency AEs, kick 1e includes only low-frequency AEs and NTMs, and kick $2 \mathrm{~d}$ includes only TAEs and EAEs. The latter three kick simulations provide further insight into which modes dominate the transport in which regions. Note, however, that to obtain a full picture it is critical to include all modes, even if they are localized far from the measurement region. This is because different modes can affect the simulation results by moving fast ions around in both position as well as velocity space.

For the kick model simulations for discharge \#153072, the effect of the varying mode amplitudes on the positive-pitch fast-ion populations at $R \approx$ $190 \mathrm{~cm}$ and $R \approx 203 \mathrm{~cm}$ is shown in figure 16 . The figure gives the energy spectra obtained by integrating the $2 \mathrm{D}$ velocity distributions over pitch for $0.3<$ $p<0.7$ for the reconstructions from measurements obtained during discharge \#153072, the corresponding neoclassical TRANSP/NUBEAM distributions, and the kick model simulations listed in table 4 .

The positive-pitch energy spectra for the reconstructions, neoclassical TRANSP/NUBEAM distribution and the kick model simulations presented in figure 15 are shown in panels a $(R \approx 190 \mathrm{~cm})$ and $\mathrm{e}$ $(R \approx 203 \mathrm{~cm})$ of figure 16 . Here, the similar trends but different densities of the two kick model simulations is easily observed: both simulations capture the hollow structure of the positive-pitch energy spectrum at $\sim 50 \mathrm{keV}$, above the half injection energy at $\sim 40 \mathrm{keV}$, that is observed in all reconstructions from measurements at $R \approx 190 \mathrm{~cm}$. Note that this positive gradient towards the injection energy in the fast-ion density for $0.3<p<0.7$ almost always appears. However, this feature is much less pronounced in the neoclassical case. The kick model simulation using the reference amplitudes (kick 1a) significantly overestimates the density decrease, whereas a better quantitative agreement between reconstructions and simulation is achieved by scaling down the amplitudes of all modes (kick 2a).

A better quantitative agreement between reconstructions from measurements and simulation can also be obtained for $R \approx 190 \mathrm{~cm}$ by reducing the experimentally uncertain low-frequency mode amplitudes whilst keeping the more certain high-frequency amplitudes at the nominal level (kick 1b-1c). This is evident from figure $16 \mathrm{p}$ that gives the positive-pitch energy spectra for the scan over low-frequency amplitudes at $R \approx 190 \mathrm{~cm}$. At $R \approx 203 \mathrm{~cm}$, on the other hand, decreasing the amplitudes of the low-frequency modes has a smaller effect on the distribution (figure 16:).

Contrarily to the scan over low-frequency modes, scanning over high-frequency mode amplitudes (figure 16 , f) only has a small, however non-negligible, effect on the fast-ion density for $0.3<p<0.7$ at both $R \approx 190 \mathrm{~cm}$ and $R \approx 203 \mathrm{~cm}$ within our amplitude limits. Interestingly, though, increasing the high-frequency mode amplitudes has the opposite effect on the fast-ion densities in the target regions at $R \approx 190 \mathrm{~cm}$ and $R \approx 203 \mathrm{~cm}$. 
Table 4. Description of kick model simulations used for 14 figure 15 and 16 and average ratios of modelled to measured neutron rates for $t=2.7-3.5 \mathrm{~s}$.

\begin{tabular}{l|l|c} 
& Description & Neutrons \\
\hline Kick 1a & All modes (reference amplitude, table 3) & $101 \%$ \\
\hline Kick 1b & All modes, amplitudes of low-frequency modes reduced to 50\% & $109 \%$ \\
\hline Kick 1c & All modes, amplitudes of low-frequency modes reduced to 25\% & $109 \%$ \\
\hline Kick 1d & Only low-frequency AEs & $154 \%$ \\
\hline Kick 1e & Only low-frequency AEs and NTMs & $147 \%$ \\
\hline Kick 2a & All modes, amplitudes of all modes reduced to 30\% & $136 \%$ \\
\hline Kick 2b & $\begin{array}{l}\text { All modes, amplitudes of low-frequency (high-frequency) } \\
\text { modes reduced to 30\% (70\%) }\end{array}$ & $121 \%$ \\
\hline Kick 2c & $\begin{array}{l}\text { All modes, amplitudes of low-frequency (high-frequency) } \\
\text { modes reduced to 30\% (85\%) }\end{array}$ & $112 \%$ \\
\hline Kick 2d & Only TAEs and EAEs & $109 \%$ \\
\hline
\end{tabular}
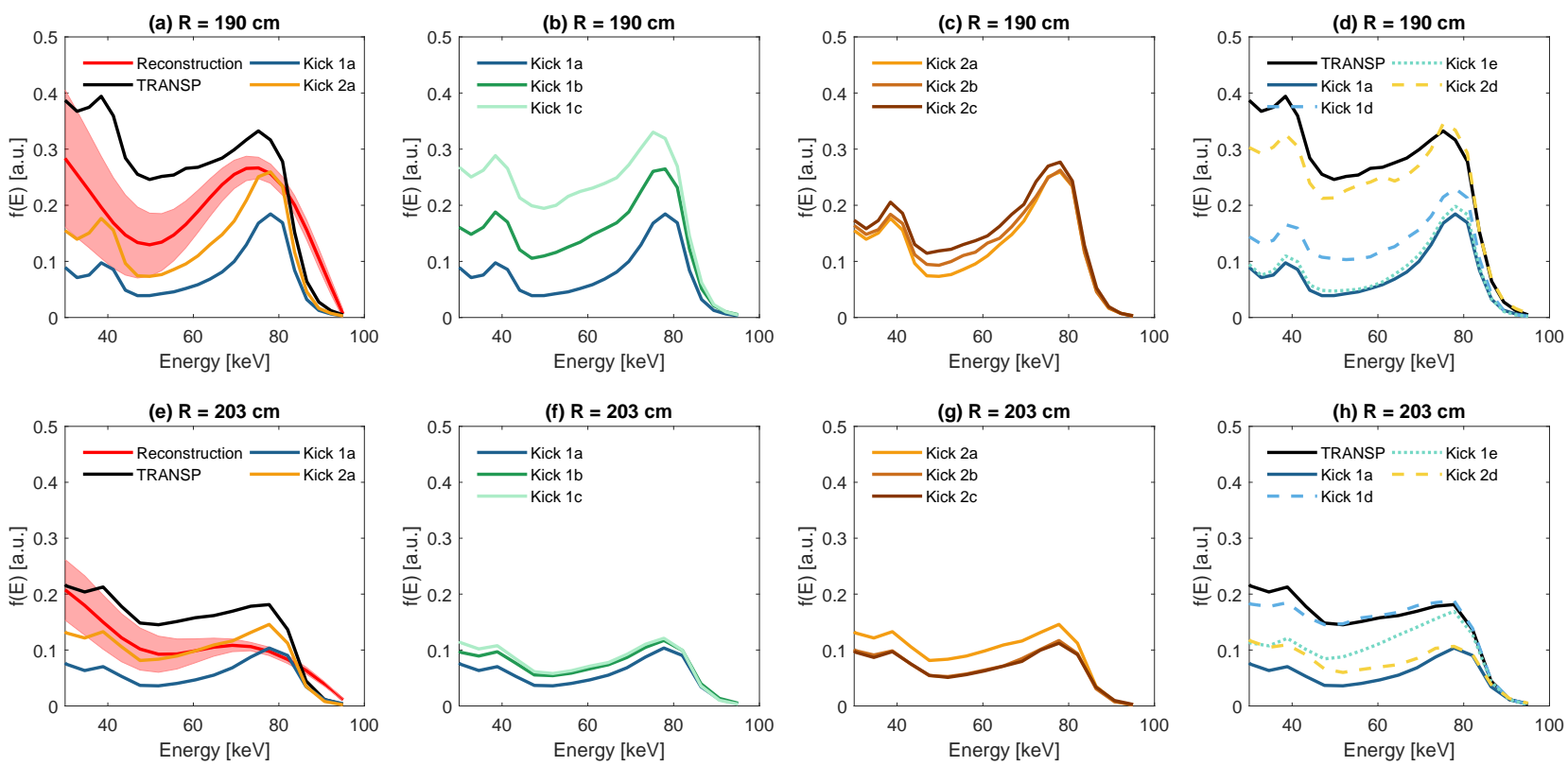

Figure 16. Energy spectra of the fast-ion distributions during discharge \#153072 obtained by integrating over pitch for $0.3<p<0.7$ for the neoclassical TRANSP/NUBEAM distribution, reconstructions from measurements, and various kick model simulations explained in table 4 at $(a-d) R \approx 190 \mathrm{~cm}$ and $(e-h) R \approx 203 \mathrm{~cm}$. In (a,e) all reconstructions from measurements (2Dbin, monotonicity-contrained, 1Dbin and simulation-prior) lie within the red-shaded areas. All spectra are scaled by the same factor as in figure 4.

The extreme cases where only some of the modes are retained (figure 16, h) suggest that the lowfrequency AEs are a dominant player in the increased transport from $R \approx 190 \mathrm{~cm}$, but have only a minor effect on the fast ions at $R \approx 203 \mathrm{~cm}$, as also expected from the scan over low-frequency amplitudes. Comparing the simulation with only low-frequency AEs (Kick 1d) to the one with only low-frequency AEs and NTMs, additionally suggests that the NTMs affect the positive-pitch distribution at both $R \approx 190 \mathrm{~cm}$ and $R \approx 203 \mathrm{~cm}$. For the extreme case retaining only the high-frequency modes, the simulation shows increased transport of positive-pitch ions from $R \approx 203 \mathrm{~cm}$, but an almost neoclassical positive-pitch distribution is obtained for $R \approx 190 \mathrm{~cm}$. In the simplest picture, this is explained by the location of the high-frequency modes (figure 13 and the fact that at $R \approx 190 \mathrm{~cm}$, only fast ions with negative pitches, i.e. outside our velocity-space target area, will have orbits overlapping these modes. This is illustrated in figure 17 that shows the projection into the poloidal plane of three test orbits intersecting the measurement volume at $R \approx 190 \mathrm{~cm}$.

The computed neutron rates for the simulations including all modes (kick 1a-1c and 2a-2c) mostly match the measured neutron rate within typical 


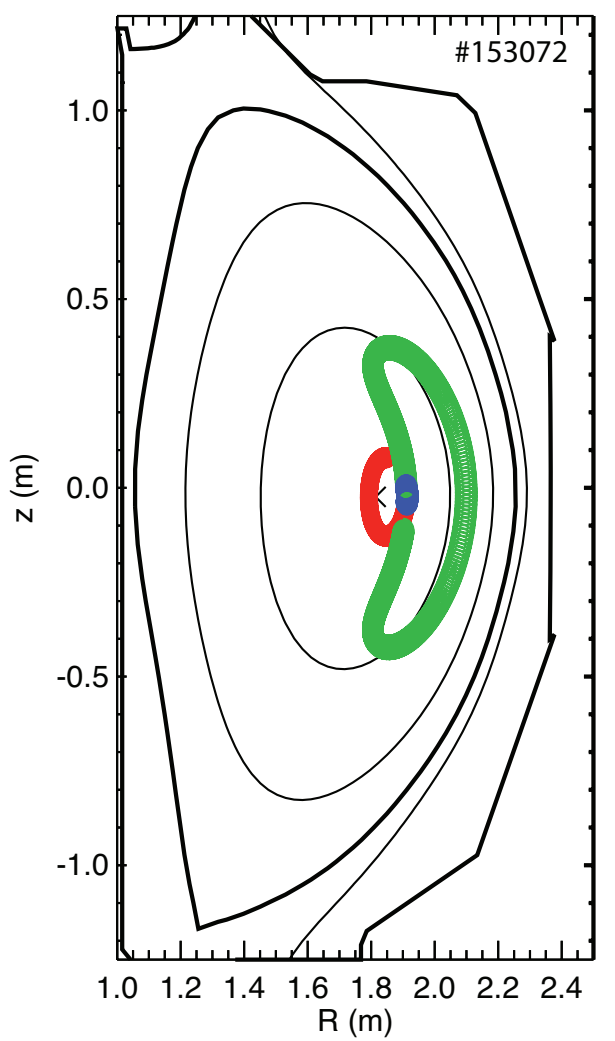

Figure 17. Elevation of DIII-D showing the projection of three $60 \mathrm{keV}$ orbits launched near $R=191 \mathrm{~cm}$ in an equilibrium from shot \#153072. The trapped orbit (green) has initial toroidal velocity $v_{p h i} / v=-0.25$, the co-passing orbit (red) has initial toroidal velocity $v_{p h i} / v=0.6$, and the stagnation orbit (blue) has initial toroidal velocity $v_{p h i} / v=$ 0.20. The magnetic axis (x), representative flux surfaces (thin lines), and last-closed flux surface and vessel boundary (thick lines) are also shown.

experimental uncertainties (not true for kick 2a). For the scan over the high-frequency mode amplitudes, reduced amplitude results in increased neutron rate, whilst the neutron rate stays almost constant for the scan over the low-frequency mode amplitudes. On the contrary, for the neoclassical TRANSP/NUBEAM simulation and for the kick model simulation in which only the lower-frequency modes (kick $1 \mathrm{~d}$ and 1e) are retained, the computed neutron rates are larger than experimentally measured. Hence, whereas the increased low-frequency mode amplitudes strongly affect the local fast-ion population at $R \approx 190 \mathrm{~cm}$, they do not significantly change the global beam-target neutron production. On the other hand, the highfrequency modes, located mainly in the outer plasma region, do not drastically affect the positive-pitch fastion distribution closer to the center, but are predicted to control the overall fast-ion loss.

The constant neutron rate but increased fast-ion transport in the central measurement volume with increased low-frequency mode amplitudes is explained by the core-localization of the NTMs (figure 13) that causes redistribution of fast ions from the center out to $\rho \sim 0.4-0.5$ without substantially changing the fastion loss rate. This is supported by the radial density profiles for the kick model simulations retaining all modes (kick 1a), only low-frequency AEs and NTMs (kick 1e), and only high-frequency modes (kick 2d) in figure 18. Note that figure 18 gives the density for all pitches whereas the energy spectra in figure 16 are computed for only $0.3<p<0.7$. In figure $16 \mathrm{~d}$, the reduced density for $0.3<p<0.7$ in kick 1e compared to the neoclassical TRANSP/NUBEAM distribution at $R \approx 203 \mathrm{~cm}$ is accompanied by density increases relative to the neoclassical distribution in other regions of velocity space, and is, hence, not reflected in figure 18

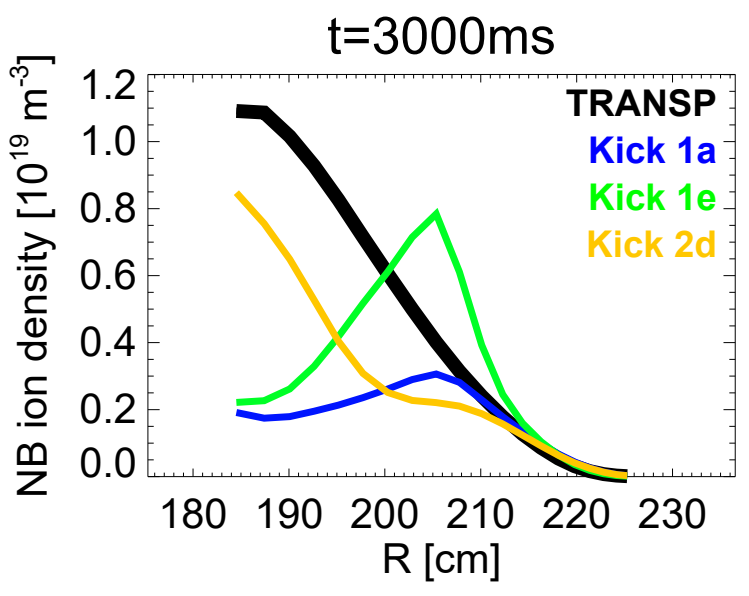

Figure 18. Radial density profile of NB ions over the entire velocity space for the kick model simulations retaining all modes (kick 1a), only low-frequency AEs and NTMs (kick 1e), and only high-frequency modes (kick 2d).

The different responses of the various modes on fast ions with different velocity-space coordinates can be understood by looking at examples of the kinetic Poincaré and transport probability matrices used for the TRANSP + kick model simulations in figure 19. Panels a-c give information about the 2/1 NTMs, whereas panels d-f and g-i relate to the low-frequency AEs and $n=4$ TAEs, respectively. The transport probability matrices represent the entire orbits and show localized phase-space regions with enhanced transport, corresponding to resonances between each instability and energetic particles. The matrices are given as functions of the fast ion energy $E$, normalized canonical toroidal momentum, $P_{\zeta} / \Psi_{w}$ where $\Psi_{w}$ is the poloidal flux at the last closed flux surface, and normalized magnetic moment, $\mu B_{0} / E$. In panels a, $\mathrm{d}$, and $\mathrm{g}$, they are shown for a constant fast-ion energy of $E=60 \mathrm{keV}$, whereas panels $\mathrm{b}$, e, and $\mathrm{h}$ are for 
a constant magnetic moment of $\mu B_{0} / E=0.2$. The location of the three test orbits shown in figure 17 are plotted as diamonds in figure $19 \mathrm{k}, \mathrm{d}$ and $\mathrm{g}$ to guide the eye, and regions with trapped, co-passing and counterpassing fast-ions are marked in panel a together with the location of the magnetic axis and the plasma edge.

For the 2/1 NTM, a single resonance dominates the interaction with fast ions. Depending on the neutral beam ion energy, the resonance is located from mid-radius inward (the $q=2$ surface is close to the magnetic axis at $\rho \approx 0.2$ ). Only trapped particles whose orbits approach the magnetic axis are affected by the perturbation. Alfvénic modes show a richer structure in terms of number of poloidal harmonics for a given toroidal mode number. Combined with a broad radial structure, more resonances are accessible to fast ions from regions near the magnetic axis to the plasma edge. In general, AE-induced transport appears larger for particles with reduced $\mu$, i.e. for copassing particles with pitches close to 1 . The effects on trapped particles depend on the mode properties (i.e. mode number spectrum and frequency), with lowfrequency AEs causing little transport while some of the TAE/EAEs induce larger kicks.

\section{Conclusion}

This study has provided the first tomographic reconstructions of the $2 \mathrm{D}$ fast-ion velocity distribution in the core plasma of the DIII-D tokamak using the four-view FIDA diagnostics. This diagnostic setup differs from similar systems at e.g. the ASDEX Upgrade and EAST tokamaks by providing only onesided spectra and by having measurement volumes with the same $(R, z)$-coordinates at different toroidal locations. This affects the calibration of the four views and causes that the negative-pitch region of velocity space is observed by only one view, making reconstructions unreliable for $p<0$.

Despite the limited diagnostic coverage for negative pitches, we found that the part of the population with positive pitches can be reconstructed convincingly. The neoclassical TRANSP/NUBEAM simulations of the fast-ion velocity distributions during the studied discharges suggested that the majority of the fast ions were co-going and that the population was monotonically increasing with pitch for $p<0$. Employing a monotonicity feature as a constraint for negative pitches, suppressed clear artefacts that appeared when reconstructing from a synthetic signal based on the neoclassical TRANSP/NUBEAM distribution for all pitches. Alternatively, reconstructing the fast-ion distribution for only positive pitches reduced the problem size and circumvented reconstructing regions with large uncertainties. We suggested two new methods for doing so: either by using the TRANSP/NUBEAM simulation for negative pitches as prior information, or by assuming a functional behaviour with pitch of the distribution in the negative-pitch region.

Using these methods, we reliably reconstructed the positive-pitch region of the fast-ion distribution at two radial positions during two DIII-D discharges with weak and strong AE and NTM activity, respectively. Using the discharge with weak activity as a reference and calibration shot, we found that the reconstructions from measurements during the discharge with high mode activity strongly disagreed with the neoclassically predicted distributions, but could, simultaneously at both measurement locations, be qualitatively well reproduced by kick model simulations where the mode activity was taken into consideration when computing the distribution. Both high- (EAEs and TAEs) and low-frequency (NTMs and unidentified low-frequency AEs) mode activity were present during the discharge. A sensitivity scan of the kick model simulations showed that the observed non-neoclassical transport of positive-pitch ions from the centermost measurement volume at $R \approx 190 \mathrm{~cm}$ was most probably predominantly caused by the lowfrequency AEs and NTMs, whereas the TAEs and EAEs became important for the transport from the measurement volume farther out at $R \approx 203 \mathrm{~cm}$, in agreement with the mode location and fast-ion orbits. In the simplest picture, this was explained by the NTMs causing redistribution of ions from the center, whilst the high-frequency modes were responsible for the fast-ion loss.

Many open questions concerning the nature of the low-frequency modes, however, still remain. These include clear mode identification and amplitude estimation, which was not well-known from experimental measurements. Also, the effects of intermittency in the mode amplitude on fast-ion transport were not addressed in the simulations and the mode amplitudes were fixed. The lack of information on radial structure and damping rate for the low-frequency AEs precludes more sophisticated modeling of their time dependent evolution. These points are, however, outside the scope of this work and are left for a future study.

The introduced inversion methods can additionally prove useful in other fast-ion velocity-space tomography studies. Using monotonicity as a constraint in either pitch or energy might prove a useful method when reconstructing the fast-ion distribution in future fusion reactors with a large population of fusionborn alpha-particles such as ITER [31], whereas the restricted velocity-space inversion methods might be relevant for the two-view FIDA systems at the MAST [16], EAST [53], TCV [54 and NSTX 55] tokamaks, 
(a)
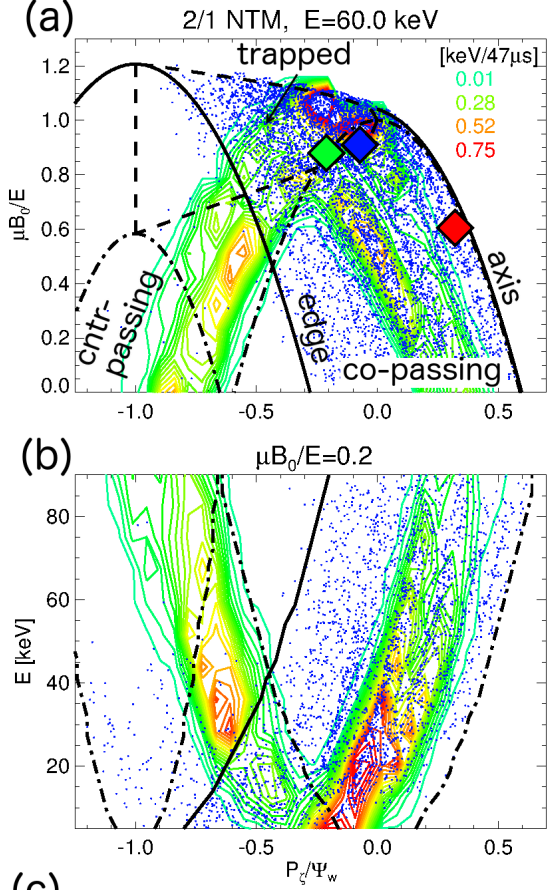

(c)

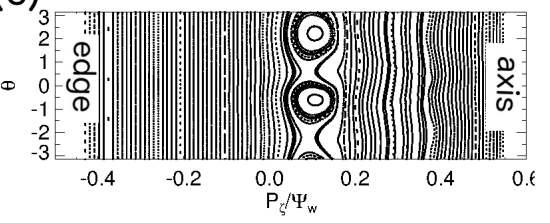

(d)

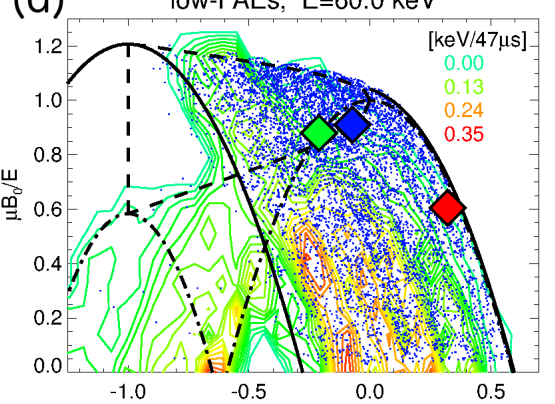

(e)

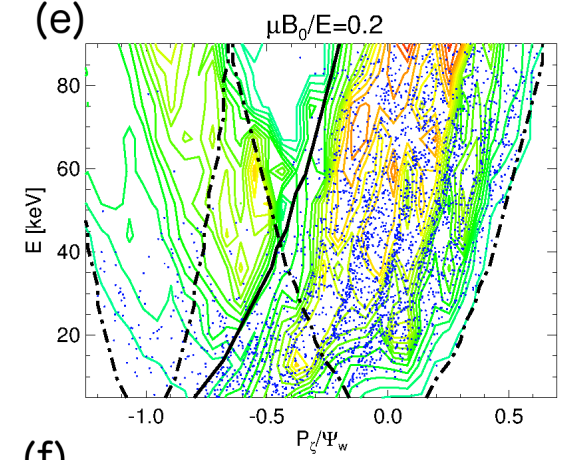

(g) $\quad n=4 T A E, E=60.0 \mathrm{keV}$

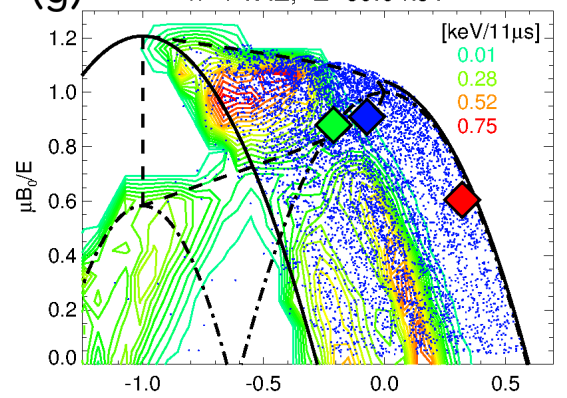

(h)

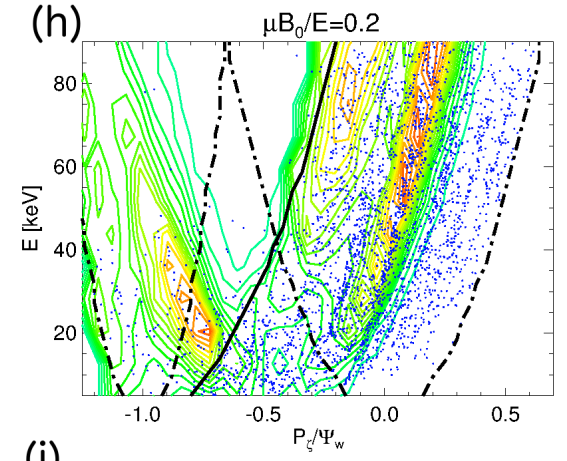

(i)

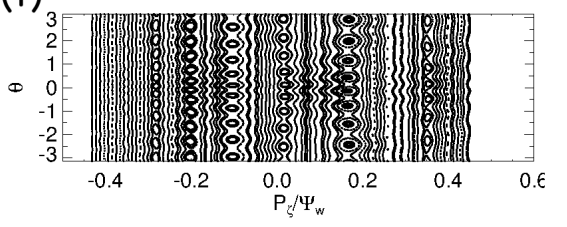

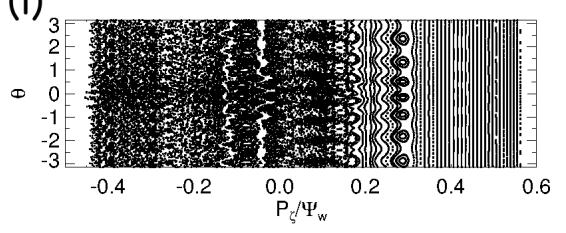

Figure 19. $(a, d, g)$ Cuts of the kick model transport probabilities at constant fast ion energy as a function of normalized canonical toroidal momentum, $P_{\zeta} / \Psi_{w}$, and magnetic moment, $\mu B_{0} / E$. Contours show the average energy kicks for the 2/1 NTM, the low-frequency AEs and an $n=4$ TAE, with red bands denoting phase-space regions with enhanced transport by instabilities. The blue dots represent a fast-ion sample from the TRANSP + kick model simulation with all instabilities active. Diamonds show the phase-space location of representative orbits intersecting the regions sampled by FIDA at $R \approx 191 \mathrm{~cm}$, using the same color-coding as in figure 17. Black lines delimit phase-space boundaries for different orbit types. (b,e,h) Average energy kicks at constant $\mu B_{0} / E=0.2$ as a function of energy and $P_{\zeta} / \Psi_{w}$. Note the different energy dependence of the kicks for these parameters, with larger kicks at lower (higher) energy for the NTM (AEs). (c,f,i) Kinetic Poincaré plots showing fast-ion resonances versus $P_{\zeta} / \Psi_{w}$ and poloidal angle $\theta$ for the three types of instabilities computed for $E \approx 60 \mathrm{keV}$ and constant $\mu B_{0} / E=0.5$.

as well as inversions based on neutron and gamma-ray spectroscopy at JET 30 .

\section{Acknowledgments}

We appreciate the support of the ITPA Topical Group for Energetic Particle Physics and the DIII$\mathrm{D}$ team. This work has been carried out within the framework of the EUROfusion Consortium and has received funding from the Euratom research and training programme 2014-2018 and 2019-2020 under grant agreement No 633053. The views and opinions expressed herein do not necessarily reflect those of the European Commission. This material is based upon work supported by the U.S. Department of Energy, Office of Science, Office of Fusion Energy Sciences, using the DIII-D National Fusion Facility, a DOE Office of Science user facility, under Award DE-FC0204ER54698, and by the U.S. Department of Energy, Office of Science, Office of Fusion Energy Sciences, Contract Number DE-AC02-09CH11466. DIII-D data shown in this paper can be obtained in digital format by following the links at https://fusion.gat.com/ global/D3D_DMP.

\section{References}

[1] W. W. Heidbrink et al. Phys. Plasmas, 15:055501, 2008.

[2] L. Chen and F. Zonca. Rev. Mod. Phys., 88:015008, 2016.

[3] M. Garcia-Munoz et al. Nucl. Fusion, 51:103013, 2011.

[4] N. N. Gorelenkov et al. Nucl. Fusion, 54(12):125001, 2014.

[5] S. D. Pinches et al. Phys. Plasmas, 22(2):021807, 2015.

[6] S. E. Sharapov et al. Nucl. Fusion, 53(10):104022, 2013. 
[7] S. D. Pinches et al. Plasma Phys. Control. Fusion, 46:B187, 2004.

[8] M. Salewski et al. Nucl. Fusion, 52:103008, 2012.

[9] M. Salewski et al. Nucl. Fusion, 53:063019, 2013.

[10] M. Salewski et al. Nucl. Fusion, 54:023005, 2014.

[11] D. Moseev et al. Rev. Mod. Plasma Phys., 2:7, 2018.

[12] B. Geiger et al. Nucl. Fusion, 55:083001, 2015.

[13] M. Weiland et al. Plasma Phys. Control. Fusion, 58:025012, 2016.

[14] M. Salewski et al. Nucl. Fusion, 56:106024, 2016.

[15] A. S. Jacobsen et al. Plasma Phys. Control. Fusion, 58:045016, 2016.

[16] B. Madsen et al. Rev. Sci. Instrum., 89:10D125, 2018.

[17] A. S. Jacobsen et al. Plasma Phys. Control. Fusion, 58:042002, 2016.

[18] W. W. Heidbrink et al. Phys. Plasmas, 24(5):056109, 2017.

[19] C. S. Collins et al. Nucl. Fusion, 57(8):086005, 2017.

[20] W. W. Heidbrink et al. Plasma Phys. Control. Fusion, 56:095030, 2014.

[21] C. T. Holcomb et al. Phys. Plasmas, 22(5):055904, 2015.

[22] M. Podestà et al. Plasma Phys. Control. Fusion, 56(5):055003, 2014.

[23] M. Podestà et al. Nucl. Fusion, 56(11):112005, 2016.

[24] M Podestà et al. Plasma Phys. Control. Fusion, 59(9):095008, 2017.

[25] M. Salewski et al. Fusion Sci. Tech., 74:23-36, 2018.

[26] W. W. Heidbrink et al. Plasma Phys. Control. Fusion, 46:1855, 2004.

[27] Y. Luo et al. Rev. Sci. Instrum., 78:033505, 2007.

[28] C. M. Muscatello et al. Rev. Sci. Instrum., 81:10D316, 2010.

[29] M. Weiland et al. Nucl. Fusion, 57:116058, 2017.

[30] M. Salewski et al. Nucl. Fusion, 57:056001, 2017.

[31] M. Salewski et al. Nucl. Fusion, 58:096019, 2018.

[32] W. W. Heidbrink et al. Phys. Rev. Lett., 71:855, 1993.

[33] N. N. Gorelenkov et al. Phys. Plasmas, 16(5):056107, 2009.

[34] H. H. Duong et al. Nucl. Fusion, 33(5):749-765, 1993.

[35] A. Pankin et al. Comput. Phys. Commun., 159:157, 2004.

[36] M. Salewski et al. Nucl. Fusion, 58:036017, 2018.

[37] W. W. Heidbrink et al. Rev. Sci. Instrum., 81:10D727, 2010.

[38] M. Podestà et al. Rev. Sci. Instrum., 79(10):10E521, 2008.

[39] Y. M. Hou et al. Rev. Sci. Instrum., 87:11E552, 2016.

[40] J. Zhang et al. Rev. Sci. Instrum., 89:10D121, 2018.

[41] W. W. Heidbrink et al. Commun. Comput. Phys., 10:716741, 2011.

[42] L. Stagner, B. Geiger, and W. W. Heidbrink. FIDASIM: A Neutral Beam and Fast-ion Diagnostic Modeling Suite. https://doi.org/10.5281/zenodo.1341369

[43] M. Salewski et al. Plasma Phys. Control. Fusion, 56:105005, 2014.

[44] W. W. Heidbrink et al. Plasma Phys. Control. Fusion, 49:1457-1475, 2007.

[45] P. C. Hansen. Discrete Inverse Problems: Insight and Algorithms. SIAM, 2010

[46] A. S. Jacobsen. Methods to determine fast-ion distribution functions from multi-diagnostic measurements. $\mathrm{PhD}$ thesis, Department of Physics, Technical University of Denmark, 2015

[47] C. A. Michael et al. Plasma Phys. Control. Fusion, 55:095007, 2013.

[48] J. Breslau, M. Gorelenkova, F. M. Poli, J. Sachdev, and X. Yuan. TRANSP. Computer Software. USDOE Office of Science (SC), Fusion Energy Sciences (FES) (SC-24). 27 Jun. 2018. Web. doi:10.11578/dc.20180627.4.

[49] R. B. White and M. S. Chance. Phys. Fluids, 27:2455, 1984.

[50] C. Z. Cheng and M. S. Chance. J. Comput. Phys., 71(1):124 $-146,1987$.

[51] N. N. Gorelenkov et al. Phys. Plasmas, 6(7):2802-2807, 1999.
[52] E. M. Carolipio et al. Nucl. Fusion, 42(7):853-862, 2002.

[53] J. Huang et al. Rev, Sci. Instrum., 85(11):11E407, 2014.

[54] B. Geiger et al. Plasma Phys. Control. Fusion, 59(11):115002, 2017.

[55] A. Bortolon et al. Rev. Sci. Instrum., 81(10):10D728, 2010. 